\title{
Near-horizon geometry and warped conformal symmetry
}

\author{
Hamid Afshar, ${ }^{a, b}$ Stéphane Detournay, ${ }^{c}$ Daniel Grumiller $^{d}$ and Blagoje Oblak ${ }^{c, e}$ \\ ${ }^{a}$ Van Swinderen Institute for Particle Physics and Gravity, University of Groningen, \\ Nijenborgh 4, 9747 AG Groningen, The Netherlands \\ ${ }^{b}$ School of Physics, Institute for Research in Fundamental Sciences (IPM), \\ P.O.Box 19395-5531, Tehran, Iran \\ ${ }^{c}$ Physique Théorique et Mathématique, Université Libre de Bruxelles and \\ International Solvay Institutes, \\ Campus Plaine C.P. 231, B-1050 Bruxelles, Belgium \\ ${ }^{d}$ Institute for Theoretical Physics, TU Wien, \\ Wiedner Hauptstrasse 8-10/136, A-1040 Vienna, Austria \\ ${ }^{e}$ DAMTP, Centre for Mathematical Sciences, University of Cambridge, \\ Wilberforce Road, Cambridge CB3 0WA, United Kingdom \\ E-mail: afshar@ipm.ir, sdetourn@ulb.ac.be, \\ grumil@hep.itp.tuwien.ac.at, boblak@ulb.ac.be
}

ABSTRACT: We provide boundary conditions for three-dimensional gravity including boosted Rindler spacetimes, representing the near-horizon geometry of non-extremal black holes or flat space cosmologies. These boundary conditions force us to make some unusual choices, like integrating the canonical boundary currents over retarded time and periodically identifying the latter. The asymptotic symmetry algebra turns out to be a Witt algebra plus a twisted $u(1)$ current algebra with vanishing level, corresponding to a twisted warped CFT that is qualitatively different from the ones studied so far in the literature. We show that this symmetry algebra is related to BMS by a twisted Sugawara construction and exhibit relevant features of our theory, including matching micro- and macroscopic calculations of the entropy of zero-mode solutions. We confirm this match in a generalization to boosted Rindler-AdS. Finally, we show how Rindler entropy emerges in a suitable limit.

Keywords: Black Holes, Chern-Simons Theories, Conformal and W Symmetry, Gaugegravity correspondence

ArXIV EPRINT: 1512.08233 


\section{Contents}

1 Introduction 2

2 Boosted Rindler boundary conditions 4

2.1 Boundary conditions 5

$\begin{array}{ll}2.2 & \text { Variational principle }\end{array}$

$\begin{array}{lll}2.3 & \text { Asymptotic symmetry transformations } & 7\end{array}$

3 Asymptotic symmetry group $\quad 8$

3.1 An empty theory 9

$\begin{array}{ll}3.2 & \text { Quasi-Rindler currents and charges } \\ 3.30\end{array}$

$\begin{array}{lll}3.3 & \text { Warped Virasoro group and coadjoint representation } & 12\end{array}$

4 Quasi-Rindler thermodynamics $\quad 14$

$\begin{array}{lll}4.1 & \text { Modular invariance and microscopic entropy } & 14\end{array}$

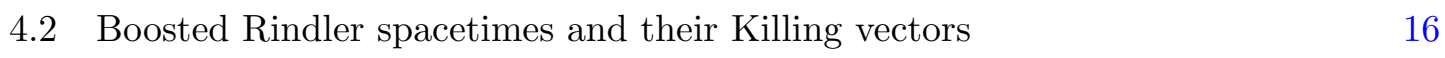

$\begin{array}{lll}4.3 & \text { Euclidean boosted Rindler } & 18\end{array}$

$\begin{array}{ll}4.4 \text { Macroscopic free energy and entropy } & 19\end{array}$

5 Boosted Rindler-AdS $\quad 20$

$\begin{array}{lll}5.1 & \text { Boundary conditions and symmetry algebra } 20\end{array}$

5.2 Microscopic quasi-Rindler-AdS entropy 22

5.3 Macroscopic quasi-Rindler-AdS entropy 22

6 Discussion 23

6.1 Role of retarded time 23

6.2 Rindler entropy? 24

$\begin{array}{lll}6.3 & \text { Other approaches } & 25\end{array}$

A On representations of the warped Virasoro group $\quad 26$

$\begin{array}{ll}\text { A.1 Coadjoint representation } & 27\end{array}$

$\begin{array}{lll}\text { A.2 Induced representations } & 30\end{array}$

B Rindler thermodynamics 33

B.1 Rindler horizon and temperature 34

$\begin{array}{lll}\text { B.2 Rindler free energy } & 35\end{array}$

$\begin{array}{lll}\text { B.3 Rindler entropy } & 35\end{array}$ 


\section{Introduction}

Rindler space arises generically as the near horizon approximation of non-extremal black holes or cosmological spacetimes. Thus, if one could establish Rindler holography one may expect it to apply universally. In particular, obtaining a microscopic understanding of Rindler entropy could pave the way towards some of the unresolved puzzles in microscopic state counting, like a detailed understanding of the entropy of the Schwarzschild black hole, which classically is one of the simplest black holes we know, but quantum-mechanically seems to be among the most complicated ones.

Our paper is motivated by this line of thought, but as we shall see the assumptions we are going to impose turn out to have a life of their own and will take us in somewhat unexpected directions. This is why we will label what we do in this work as "quasi-Rindler holography" instead of "Rindler holography".

Three-dimensional gravity. For technical reasons we consider Einstein gravity in three spacetime dimensions [1-3]. While simpler than its higher-dimensional relatives, it is still complex enough to exhibit many of the interesting features of gravity: black holes $[4,5]$, cosmological spacetimes [6, 7] and boundary gravitons [8].

In the presence of a negative cosmological constant the seminal paper by Brown and Henneaux [8] established one of the precursors of the AdS/CFT correspondence. The key ingredient to their discovery that $\mathrm{AdS}_{3}$ Einstein gravity must be dual to a $\mathrm{CFT}_{2}$ was the imposition of precise (asymptotically AdS) boundary conditions. This led to the realization that some of the bulk first class constraints become second class at the boundary, so that boundary states emerge and the physical Hilbert space forms a representation of the 2dimensional conformal algebra with specific values for the central charges determined by the gravitational theory.

As it turned out, the Brown-Henneaux boundary conditions can be modified, in the presence of matter [9], in the presence of higher derivative interactions [10, 11] and even in pure Einstein gravity $[12,13]$. In the present work we shall be concerned with a new type of boundary conditions for 3-dimensional Einstein gravity without cosmological constant. Let us therefore review some features of flat space Einstein gravity.

In the absence of a cosmological constant Barnich and Compère pioneered a BrownHenneaux type of analysis of asymptotically flat boundary conditions [14] and found a specific central extension of the $\mathrm{BMS}_{3}$ algebra [15], which is isomorphic to the Galilean conformal algebra [16-18]. Based on this, there were numerous developments in the past few years, like the flat space chiral gravity proposal [19], the counting of flat space cosmology microstates [20,21], the existence of phase transitions between flat space cosmologies and hot flat space [22, 23], higher spin generalizations of flat space gravity [24, 25], new insights into representations and characters of the $\mathrm{BMS}_{3}$ group [26-28] with applications to the 1-loop partition function [29], flat space holographic entanglement entropy [30-32] and numerous other aspects of flat space holography [33-46].

Quasi-Rindler gravity. As we shall show in this paper, the flat space Einstein equations,

$$
R_{\mu \nu}=0,
$$


do allow for solutions that do not obey the Barnich-Compère boundary conditions but instead exhibit asymptotically Rindler behavior,

$$
\mathrm{d} s^{2} \sim \mathcal{O}(r) \mathrm{d} u^{2}-2 \mathrm{~d} u \mathrm{~d} r+\mathrm{d} x^{2}+\mathcal{O}(1) \mathrm{d} u \mathrm{~d} x+\ldots
$$

The main goal of the present work is to set up these boundary conditions, to prove their consistency and to discuss some of the most relevant consequences for holography, in particular the asymptotic symmetry algebra and a comparison between macroscopic and microscopic entropies. Some previous literature on asymptotically Rindler spacetimes is provided by refs. [47-74]. For some previous literature on black hole entropy from a near horizon conformal field theory perspective see [75] and references therein.

Before we delve into the relevant technicalities we address one conceptual issue that may appear to stop any attempt to Rindler holography in its track. For extremal black holes the usual near-horizon story works due to their infinite throat, which implies that one can consistently replace the region near the horizon by the near-horizon geometry and apply holography to the latter, see e.g. section 2 in [76] for a review. By contrast, non-extremal black holes do not have an infinite throat. Therefore, the asymptotic region of Rindler space in general has nothing to do with the near-horizon region of the original theory. So even if one were to find some dual field theory in some asymptotic Rindler space, it may not be clear why the corresponding physical states should be associated with the original black hole or cosmological spacetime. However, as we shall see explicitly, the notion of a "dual theory living at the boundary" is misleading; one could equally say that the "field theory lives near the horizon", since (in three dimensions) the canonical charges responsible for the emergence of physical boundary states are independent of the radial coordinate. While we are going to encounter a couple of obstacles to apply Rindler holography the way we originally intended to do, we do not consider the finiteness of the throat of non-extremal black holes as one of them.

Starting with the assumption (1.2) we are led to several consequences that we did not anticipate. The first one is that, on-shell, the functions specifying the spacetime metric depend on retarded time $u$ instead of the spatial coordinate $x$, as do the components of the asymptotic Killing vector fields. As a consequence, canonical charges written as integrals over $x$ all vanish and the asymptotic symmetries are all pure gauge. However, upon writing surface charges as integrals over $u$ and taking time to be periodic, the asymptotic symmetry algebra turns out to describe a warped CFT of a type not encountered before: there is no Virasoro central charge nor a $u(1)$-level; instead, there is a non-trivial cocycle in the mixed commutator. Based on these results we determine the entropy microscopically and find that it does not coincide with the naive Rindler entropy, as a consequence of the different roles that $u$ and $x$ play in quasi-Rindler, versus Rindler, holography. In the quasi-Rindler setting, we show that our microscopic result for entropy does match the macroscopic one. As we shall see, the same matching occurs in our generalization to Rindler-AdS.

In summary, in this paper we describe a novel type of theory with interesting symmetries inspired by, but slightly different from, naive expectations of Rindler holography.

This work is organized as follows. In section 2 we state boosted Rindler boundary conditions and provide a few consistency checks. In section 3 we derive the asymptotic 
symmetry algebra with its central extension and discuss some implications for the putative dual theory. Then, in section 4, we address quasi-Rindler thermodynamics, calculate free energy and compare macroscopic with microscopic results for entropy, finding exact agreement between the two. Section 5 is devoted to the generalization of the discussion to quasi-Rindler AdS. Finally, we conclude in section 6 with some of the unresolved issues. Along the way, we encounter novel aspects of warped conformal field theories, which we explore in appendix A. Questions related to standard Rindler thermodynamics are relegated to appendix B.

\section{Boosted Rindler boundary conditions}

The 3-dimensional line-element $[u, r, x \in(-\infty, \infty)]$

$$
\mathrm{d} s^{2}=-2 a(u) r \mathrm{~d} u^{2}-2 \mathrm{~d} u \mathrm{~d} r+2 \eta(u) \mathrm{d} u \mathrm{~d} x+\mathrm{d} x^{2}
$$

solves the vacuum Einstein equations (1.1) for all functions $a, \eta$ that depend solely on the retarded time $u$. For vanishing $\eta$ and constant positive $a$ the line-element (2.1) describes Rindler space with acceleration $a$. [This explains why we chose the factor -2 in the first term in (2.1).] If $\eta$ does not vanish we have boosted Rindler space. These observations motivate us to formulate consistent boundary conditions that include all line-elements of the form (2.1) as allowed classical states.

The gravity bulk action we are going to use is the Einstein-Hilbert action

$$
I_{\mathrm{EH}}=\frac{k}{4 \pi} \int \mathrm{d}^{3} x \sqrt{-g} R, \quad k=\frac{1}{4 G_{N}},
$$

which, up to boundary terms, is equivalent to the Chern-Simons action $[77,78]$

$$
I_{\mathrm{CS}}=\frac{k}{4 \pi} \int\left\langle A \wedge \mathrm{d} A+\frac{2}{3} A \wedge A \wedge A\right\rangle
$$

with an $i s l(2)$ connection $A$ and corresponding invariant bilinear form $\langle\cdots\rangle$. Explicitly, the $i s l(2)$ generators span the Poincaré algebra in three dimensions $(n, m=0, \pm 1)$,

$$
\left[L_{n}, L_{m}\right]=(n-m) L_{n+m} \quad\left[L_{n}, M_{m}\right]=(n-m) M_{n+m} \quad\left[M_{n}, M_{m}\right]=0,
$$

and their pairing with respect to the bilinear form $\langle\cdots\rangle$ is

$$
\left\langle L_{1}, M_{-1}\right\rangle=\left\langle L_{-1}, M_{1}\right\rangle=-2 \quad\left\langle L_{0}, M_{0}\right\rangle=1
$$

(all bilinears not mentioned here vanish). The connection can be decomposed in components as $A=A_{L}^{+} L_{1}+A_{L}^{0} L_{0}+A_{L}^{-} L_{-1}+A_{M}^{+} M_{1}+A_{M}^{0} M_{0}+A_{M}^{-} M_{-1}$. In terms of these components the line-element reads

$$
\mathrm{d} s^{2}=g_{\mu \nu} \mathrm{d} x^{\mu} \mathrm{d} x^{\nu}=-4 A_{M}^{+} A_{M}^{-}+\left(A_{M}^{0}\right)^{2} .
$$

Thus, from a geometric perspective the components $A_{M}^{n}$ correspond to the dreibein and the components $A_{L}^{n}$ to the (dualized) spin-connection $[79,80]$. 


\subsection{Boundary conditions}

In the metric formulation, boosted Rindler boundary conditions at null infinity $r \rightarrow+\infty$ are given by

$$
g_{\mu \nu}=\left(\begin{array}{ccc}
-2 a(u, x) r+\mathcal{O}(1) & -1+\mathcal{O}(1 / r) & \eta(u, x)+\mathcal{O}(1 / r) \\
g_{r u}=g_{u r} & \mathcal{O}\left(1 / r^{2}\right) & \mathcal{O}(1 / r) \\
g_{x u}=g_{u x} & g_{x r}=g_{r x} & 1+\mathcal{O}(1 / r)
\end{array}\right)
$$

where $a(u, x)$ and $\eta(u, x)$ are arbitrary, fluctuating $\mathcal{O}(1)$ functions. The equations of motion (1.1) imply homogeneity of the Rindler acceleration:

$$
\partial_{x} a(u, x)=0 .
$$

The function $\eta(u, x)$ and subleading terms are also constrained by the equations of motion. These constraints are solved by functions $\eta$ that depend on $u$ only: ${ }^{1}$

$$
\partial_{x} \eta(u, x)=0 .
$$

For simplicity, from now on we always implement the asymptotic on-shell conditions (2.8), (2.9) together with the boundary conditions (2.7), i.e., we assume both $a$ and $\eta$ depend on the retarded time $u$ only.

Since the $x$-independence of the functions $a$ and $\eta$ has important consequences we stress that the conditions (2.8)-(2.9) are forced upon us by the Einstein equations and our choice of boundary conditions (2.7). In fact, similar boundary conditions were proposed already in four dimensions [63], but no attempt to identify the dual theory was made in that paper.

For many applications it is useful to recast these boundary conditions in first order form in terms of an $i s l(2)$ connection

$$
A=b^{-1}(\mathrm{~d}+\mathfrak{a}+\mathcal{O}(1 / r)) b,
$$

with the ISL(2) group element

$$
b=\exp \left(\frac{r}{2} M_{-1}\right)
$$

and the auxiliary connection

$$
\begin{aligned}
\mathfrak{a}_{L}^{+} & =0 & & \mathfrak{a}_{M}^{+}=\mathrm{d} u \\
\mathfrak{a}_{L}^{0} & =a(u) \mathrm{d} u & & \mathfrak{a}_{M}^{0}=\mathrm{d} x \\
\mathfrak{a}_{L}^{-} & =-\frac{1}{2}\left(\eta^{\prime}(u)+a(u) \eta(u)\right) \mathrm{d} u & & \mathfrak{a}_{M}^{-}=-\frac{1}{2} \eta(u) \mathrm{d} x
\end{aligned}
$$

where \pm refers to $L_{ \pm 1}$ and $M_{ \pm 1}$. Explicitly,

$$
A=\mathfrak{a}+\frac{\mathrm{d} r}{2} M_{-1}+\frac{a(u) r \mathrm{~d} u}{2} M_{-1}
$$

\footnotetext{
${ }^{1}$ The most general solution of these constraints (up to gauge transformations) is actually $\eta(u, x)=$ $\eta(u)+k(x) \exp \left[-\int^{u} a\left(u^{\prime}\right) \mathrm{d} u^{\prime}\right]$. We considered this more general case but did not find relevant new features when $k(x)$ is non-zero. In particular, the function $k(x)$ does not contribute to the canonical surface charges, so we set it to zero with no loss of generality.
} 
where the second term comes from $b^{-1} \mathrm{~d} b$ and the linear term in $r$ from applying the Baker-Campbell-Hausdorff formula $b^{-1} \mathfrak{a} b=\mathfrak{a}-\frac{r}{2}\left[M_{-1}, \mathfrak{a}\right]$. Using (2.6), this Chern-Simons connection is equivalent to the metric (2.1).

\subsection{Variational principle}

For a well-defined variational principle the first variation of the full action $\Gamma$ must vanish for all variations that preserve our boundary conditions (2.7). Since we shall later employ the Euclidean action to determine the free energy we use Euclidean signature here too.

We make the ansatz ${ }^{2}$

$$
\Gamma=-\frac{1}{16 \pi G_{N}} \int \mathrm{d}^{3} x \sqrt{g} R-\frac{\alpha}{8 \pi G_{N}} \int \mathrm{d}^{2} x \sqrt{\gamma} K
$$

where $\alpha$ is some real parameter. If $\alpha=1$, we recover the Gibbons-Hawking-York action [81, 82]. If $\alpha=\frac{1}{2}$, we recover an action that is consistent in flat space holography [23]. We check now which value of $\alpha$ - if any - is consistent for our boosted Rindler boundary conditions (2.7).

Dropping corner terms, the first variation of the action (2.14) reads on-shell [23]

$$
\left.\delta \Gamma\right|_{\text {EOM }}=\frac{1}{16 \pi G_{N}} \int \mathrm{d}^{2} x \sqrt{\gamma}\left(K^{i j}-\alpha K g^{i j}+(2 \alpha-1) K n^{i} n^{j}+(1-\alpha) \gamma^{i j} n^{k} \nabla_{k}\right) \delta g_{i j} .
$$

We introduce now a cut-off at $r=r_{c}$ and place the boundary at this cut-off, with the idea of letting $r_{c} \rightarrow \infty$ at the end of our calculations. Some useful asymptotic expressions are $\left[n_{i}=\delta_{i}^{r} \frac{1}{\sqrt{2 a r_{c}}}+\mathcal{O}\left(1 / r_{c}^{3 / 2}\right)\right.$ is the outward pointing unit normal vector, $\gamma=2 a r_{c}+\mathcal{O}(1)$ the determinant of the induced metric at the boundary and $K=K^{i}{ }_{i}=\sqrt{\frac{a}{2 r_{c}}}+\mathcal{O}\left(1 / r_{c}^{3 / 2}\right)$ the trace of extrinsic curvature]:

$$
\begin{aligned}
\sqrt{\gamma} K^{i j} \delta g_{i j} & =\delta a+\mathcal{O}\left(1 / r_{c}\right) & \sqrt{\gamma} K g^{i j} \delta g_{i j} & =\mathcal{O}\left(1 / r_{c}\right) \\
\sqrt{\gamma} K n^{i} n^{j} \delta g_{i j} & =-\delta a+\mathcal{O}\left(1 / r_{c}\right) & \sqrt{\gamma} \gamma^{i j} n^{k} \nabla_{k} \delta g_{i j} & =\mathcal{O}\left(1 / r_{c}\right) .
\end{aligned}
$$

Inserting these expressions into (2.15) establishes

$$
\left.\delta \Gamma\right|_{\mathrm{EOM}}=\frac{1}{8 \pi G_{N}} \int \mathrm{d}^{2} x(1-\alpha) \delta a+\mathcal{O}\left(1 / r_{c}\right) .
$$

Therefore, picking $\alpha=1$ we have a well-defined variational principle.

Demanding a well-defined variational principle for the first order action (2.3) with the boundary conditions (2.10)-(2.12) also requires the addition of a boundary term of the form

$$
\Gamma_{\mathrm{CS}}=I_{\mathrm{CS}} \pm \frac{k}{4 \pi} \int \mathrm{d} u \mathrm{~d} x\left\langle A_{u} A_{x}\right\rangle
$$

\footnotetext{
${ }^{2}$ In the Lorentzian theory the boundary is time-like (space-like) if $a$ is positive (negative). To accommodate both signs one should replace $K$ by $|K|$. To reduce clutter we assume positive $a$ and moreover restrict to zero mode solutions, $a=$ const., $\eta=$ const. It can be shown that the variational principle is satisfied for non-zero mode solutions if it is satisfied for zero mode solutions, as long as $\partial_{x} g_{r x}=\mathcal{O}\left(1 / r^{2}\right)$. We thank Friedrich Schöller for discussions on the variational principle and for correcting a factor two.
} 
where the sign depends on the conventions for the boundary volume form, $\epsilon^{u x}=\mp 1$. This result agrees with the general expression found in three-dimensional gravity in flat space $[79,83,84]$.

In conclusion, the full Euclidean second order action suitable for quasi-Rindler holography is given by

$$
\Gamma=-\frac{1}{16 \pi G_{N}} \int \mathrm{d}^{3} x \sqrt{g} R-\frac{1}{8 \pi G_{N}} \int \mathrm{d}^{2} x \sqrt{\gamma} K
$$

where the boundary contribution is the Gibbons-Hawking-York boundary term. This action is the basis for Rindler thermodynamics discussed in section 4 .

\subsection{Asymptotic symmetry transformations}

The allowed diffeomorphisms preserving the boundary conditions (2.7) are generated by vector fields $\xi$ whose components are

$$
\begin{aligned}
& \xi^{u}=t(u)+\mathcal{O}(1 / r), \\
& \xi^{r}=-r t^{\prime}(u)+\mathcal{O}(1), \\
& \xi^{x}=p(u)+\mathcal{O}(1 / r),
\end{aligned}
$$

where $t(u)$ and $p(u)$ are arbitrary real functions. Infinitesimally, the corresponding diffeomorphisms take the form

$$
u \mapsto u+\epsilon t(u), \quad x \mapsto x+\epsilon p(u)
$$

on the spacetime boundary at infinity. In other words, the gravitational system defined by the boundary conditions (2.7) is invariant under time reparametrizations generated by $t(u) \partial_{u}-r t^{\prime}(u) \partial_{r}$ and under time-dependent translations of $x$ generated by $p(u) \partial_{x}$. These symmetries are reminiscent of those of two-dimensional Galilean conformal field theories [17].

The Lie bracket algebra of allowed diffeomorphisms (2.21) is the semi-direct sum of a Witt algebra and a $u(1)$ current algebra. This can be seen, for instance, by thinking of $u$ as a complex coordinate (which will indeed be appropriate for thermodynamical applications) and expanding $t(u)$ and $p(u)$ in Laurent modes. Another way to obtain the same result is to take $u$ periodic, say

$$
u \sim u+2 \pi L
$$

(where $L$ is some length scale), and to expand the functions $t(u)$ and $p(u)$ in Fourier modes. Introducing the generators

$$
\left.t_{n} \equiv \xi\right|_{t(u)=L e^{i n u / L}, p(u)=0} \quad \text { and }\left.\quad p_{n} \equiv \xi\right|_{t(u)=0, p(u)=L e^{i n u / L}}
$$

then yields the Lie brackets

$$
\begin{aligned}
i\left[t_{n}, t_{m}\right] & =(n-m) t_{n+m} \\
i\left[t_{n}, p_{m}\right] & =-m p_{n+m} \\
i\left[p_{n}, p_{m}\right] & =0
\end{aligned}
$$


up to subleading corrections that vanish in the limit $r \rightarrow+\infty$. Thus, quasi-Rindler boundary conditions differ qualitatively from usual AdS holography, which relies on conformal symmetry, and from flat space holography [8, 85], which relies on BMS symmetry $[14,18]$. Instead, if there exists a dual theory for quasi-Rindler boundary conditions, it should be a warped CFT [86] whose conformal symmetry is replaced by time-reparametrization invariance. ${ }^{3}$ We will return to the interpretation of this symmetry in section 3 .

The allowed diffeomorphisms (2.21) can also be obtained from the Chern-Simons formulation: upon looking for $i s l(2)$ gauge parameters $\widehat{\varepsilon}$ that obey

$$
\delta_{\widehat{\varepsilon}} A=\mathrm{d} \widehat{\varepsilon}+[A, \widehat{\varepsilon}]=\mathcal{O}(\delta A)
$$

where $\delta A$ denotes the fluctuations allowed by the boundary conditions (2.10)-(2.12), and writing

$$
\widehat{\varepsilon}=b^{-1}(\varepsilon+\mathcal{O}(1 / r)) b
$$

[in terms of the group element (2.11)], one finds

$$
\begin{aligned}
\varepsilon= & t(u) M_{1}+p(u) M_{0}+\Upsilon(u) M_{-1}+\left(a(u) t(u)-t^{\prime}(u)\right) L_{0} \\
& -\frac{1}{2}\left(\left(\eta^{\prime}(u)+a(u) \eta(u)\right) t(u)+p^{\prime}(u)\right) L_{-1} .
\end{aligned}
$$

Here the functions $t(u)$ and $p(u)$ are those of (2.21), while $\Upsilon(u)$ solves the differential equation

$$
2 \Upsilon^{\prime}(u)+2 \Upsilon(u) a(u)+p(u)\left(\eta^{\prime}(u)+a(u) \eta(u)\right)=0 .
$$

Upon imposing periodicity (2.23), this solution is unique.

Using (2.26)-(2.28) and the on-shell connection (2.12), we find that the functions $a$ and $\eta$ transform as follows under allowed diffeomorphisms:

$$
\delta a=t a^{\prime}+t^{\prime} a-t^{\prime \prime} \quad \delta \eta=t \eta^{\prime}+t^{\prime} \eta+p^{\prime} .
$$

(Prime denotes differentiation with respect to $u$.) Note in particular that translations by $p$ leave $a$ invariant; note also the inhomogeneous term $t^{\prime \prime}$ in the infinitesimal transformation law of $a$ under conformal transformations, which hints that the asymptotic symmetry algebra has a central extension. We are now going to verify this.

\section{Asymptotic symmetry group}

This section is devoted to the surface charges associated with the asymptotic symmetries (2.21). First we show that the conventional approach leads to a trivial theory where all asymptotic symmetries are gauge transformations, as any on-shell metric is gaugeequivalent to Minkowski space. We then opt in subsection 3.2 for a non-standard definition of surface charges, providing us with a centrally extended asymptotic symmetry algebra. In subsection 3.3 we work out the finite transformations of the dual energy-momentum tensor.

\footnotetext{
${ }^{3}$ Warped CFT symmetry algebras have appeared in the context of Topologically Massive Gravity [87] (see also [88-90]), Lobachevsky holography [91], conformal gravity with generalized AdS or flat boundary conditions [80, 92, 93], Lower Spin Gravity [94], and Einstein gravity [12, 13]. On the field theory side these symmetries were shown to be a consequence, under certain conditions, of translation and chiral scale invariance [95].
} 


\subsection{An empty theory}

We saw in the previous section that asymptotic symmetries include time reparametrizations. This is a somewhat ambiguous situation: on the one hand, asymptotic symmetries are generally interpreted as global symmetries, but on the other hand, time reparametrizations are usually seen as gauge symmetries. In this subsection we show how the standard approach to surface charges in gravity selects the latter interpretation.

In the Chern-Simons formulation [79, 80, 83, 84, 96, 97], the variation of the canonical current $\mathfrak{j}$ associated with an asymptotic symmetry generated by $\widehat{\varepsilon}$ reads

$$
\delta \mathrm{j}[\varepsilon]=\frac{k}{2 \pi}\langle\widehat{\varepsilon}, \delta A\rangle=\frac{k}{2 \pi}\langle\varepsilon, \delta \mathfrak{a}\rangle,
$$

where $A$ and $\mathfrak{a}$ are related by (2.10) and $\langle\cdots\rangle$ denotes the invariant bilinear form (2.5). The integral of that expression along a line or a circle at infinity gives the variation of the corresponding surface charge [98-100]. In the present case, the region $r \rightarrow+\infty$ is spanned by the coordinates $u$ and $x$, but only the latter is space-like. ${ }^{4}$ Accordingly, the natural surface charges are integrals over $x$; unfortunately the boundary conditions (2.12) [and the ensuing asymptotic symmetries (2.28)] set to zero the $x$-component of the variation (3.1), so that these charges all vanish. It follows that, from this viewpoint, all asymptotic symmetries are in fact gauge symmetries; there are no global symmetries whatsoever, and the theory is empty.

While this conclusion is somewhat disappointing, it does not prevent us from studying the group of gauge symmetries in its own right, and these considerations will in fact be useful once we turn to an alternative interpretation. Upon integrating the infinitesimal transformations (2.22), one obtains finite diffeomorphisms of the plane $\mathbb{R}^{2}$ (spanned by the coordinates $u$ and $x$ ) given by

$$
u \mapsto f(u), \quad x \mapsto x+p(f(u)),
$$

where $f$ is an orientation-preserving diffeomorphism of the real line (so that $f^{\prime}(u)>0$ for all $u$ ), and $p$ is an arbitrary function. Such pairs $(f, p)$ span a group

$$
G \equiv \operatorname{Diff}(\mathbb{R}) \ltimes \mathrm{C}^{\infty}(\mathbb{R}),
$$

where the vector space $\mathrm{C}^{\infty}(\mathbb{R})$ is seen as an Abelian group with respect to pointwise addition. Diffeomorphisms act on functions according to

$$
\left(\sigma_{f} p\right)(u) \equiv p\left(f^{-1}(u)\right), \quad \text { i.e. } \quad \sigma_{f} p \equiv p \circ f^{-1},
$$

so $G$ is a semi-direct product with a group operation

$$
\left(f_{1}, p_{1}\right) \cdot\left(f_{2}, p_{2}\right) \equiv\left(f_{1} \circ f_{2}, p_{1}+\sigma_{f_{1}} p_{2}\right) .
$$

It is a centerless version of the symmetry group of warped conformal field theories.

\footnotetext{
${ }^{4}$ Note that the radial dependence captured by the group element $b$ defined in (2.11) drops out of the canonical currents (3.1). The corresponding charges can therefore be defined on any $r=$ const. slice (including the horizon).
} 
One may then ask how finite gauge transformations affect the on-shell metrics (2.1), given that the infinitesimal transformations are (2.30). We show in appendix A.1 that, under the action of a gauge transformation $(f, p)$, the functions $\eta(u)$ and $a(u)$ are mapped on new functions $\tilde{\eta}$ and $\tilde{a}$ given by

$$
\tilde{\eta}(f(u))=\frac{1}{f^{\prime}(u)}\left[\eta(u)-(p \circ f)^{\prime}(u)\right], \quad \tilde{a}(f(u))=\frac{1}{f^{\prime}(u)}\left[a(u)+\frac{f^{\prime \prime}(u)}{f^{\prime}(u)}\right] .
$$

It is easily verified that these transformations reduce to (2.30) upon taking $f(u)=u+\epsilon t(u)$, replacing $p(u)$ by $\epsilon p(u)$ and expanding to first order in $\epsilon$. This formula shows explicitly that the phase space of the theory is empty, as any diffeomorphism $f$ such that

$$
f^{\prime}(u)=C \exp \left[-\int_{0}^{u} a(v) \mathrm{d} v\right], \quad C>0
$$

maps $a(u)$ on $\tilde{a}=0$. When combining this map with a suitable translation $p(u)$, the whole metric (2.1) is mapped on that of Minkoswki space, so that indeed any solution is pure gauge. Note that the inhomogeneous term proportional to $f^{\prime \prime} / f^{\prime}$ in the transformation law of $a$ is crucial in order for the latter statement to be true.

In principle one can impose suitable fall-off conditions on the functions $a(u)$ and $\eta(u)$ at future and past infinity, and study the subgroup of (3.3) that preserves these conditions. For example, $a(u) \sim$ const. $+\mathcal{O}(1 /|u|)$ would include Rindler spacetime, potentially leading to interesting asymptotic symmetries at $|u| \rightarrow+\infty$. We will not follow this approach here; instead, we will try to make the theory non-trivial by using an unconventional prescription for the definition of surface charges.

\subsection{Quasi-Rindler currents and charges}

In the previous subsection we interpreted asymptotic symmetries as gauge symmetries, in accordance with the fact that all surface charges written as integrals over a space-like slice at infinity vanish. However, another interpretation is available: instead of integrating (3.1) over $x$, we may decide to integrate it over retarded time $u$. Despite clashing with the usual Hamiltonian formalism, this approach is indeed the most natural one suggested by the $u$-dependent asymptotic Killing vector fields (2.21) and the solutions (2.1).

For convenience we will also assume that the coordinate $u$ is periodic as in (2.23). This condition introduces closed time-like curves and breaks Poincaré symmetry (even when $a=0$ !); it sets off our departure from the world of Rindler to that of quasi-Rindler holography. While it seems unnatural from a gravitational/spacetime perspective, this choice is naturally suggested by our asymptotic symmetries and our phase space. In the remainder of this paper we explore its consequences, assuming in particular that the functions $t(u)$, $p(u), \eta(u)$ and $a(u)$ are $2 \pi L$-periodic. This will in fact lead us to study new aspects of warped conformal field theories, which we believe are interesting in their own right. 
In the quasi-Rindler case, the variation of the surface charge associated with the symmetry transformation $(t, p)$, evaluated on the metric $(a, \eta)$, reads

$$
\delta Q_{(a, \eta)}[t, p]=\int_{0}^{2 \pi L} \mathrm{~d} u \delta \mathbf{j}_{u} .
$$

Using (3.1) and inserting expressions (2.12) and (2.28) yields

$$
\delta Q_{(a, \eta)}[t, p]=\frac{k}{2 \pi} \int_{0}^{2 \pi L} \mathrm{~d} u(t(u) \delta T(u)+p(u) \delta P(u)),
$$

where

$$
T(u)=\eta^{\prime}(u)+a(u) \eta(u), \quad P(u)=a(u),
$$

so that the charges are finite and integrable:

$$
Q_{(a, \eta)}[t, p]=\frac{k}{2 \pi} \int_{0}^{2 \pi L} \mathrm{~d} u(t(u) T(u)+p(u) P(u)) .
$$

This expression shows in particular that the space of solutions $(a, \eta)$ is dual to the asymptotic symmetry algebra ${ }^{5}[26,27,101-103]$. More precisely, the pair of functions $(T(u), P(u))$ transforms under the coadjoint representation of the asymptotic symmetry group, with $T(u)$ dual to time reparametrizations and $P(u)$ dual to translations of $x$. This observation will be crucial when determining the transformation law of $T(u)$ and $P(u)$ under finite asymptotic symmetry transformations, which in turn will lead to a Cardy-like entropy formula.

From the variations $(2.30)$ of the functions $(a, \eta)$, we deduce corresponding variations of the functions $T$ and $P$ in (3.10):

$$
\delta_{(t, p)} P=t P^{\prime}+t^{\prime} P-t^{\prime \prime}, \quad \delta_{(t, p)} T=t T^{\prime}+2 t^{\prime} T+p^{\prime} P+p^{\prime \prime} .
$$

This result contains all the information about the surface charge algebra, including its central extensions. On account of $2 \pi L$-periodicity in the retarded time $u$, we can introduce the Fourier-mode generators

$$
T_{n} \equiv \frac{k L}{2 \pi} \int_{0}^{2 \pi L} \mathrm{~d} u e^{i n u / L} T(u) \quad P_{n} \equiv \frac{k}{2 \pi} \int_{0}^{2 \pi L} \mathrm{~d} u e^{i n u / L} P(u),
$$

whose Poisson brackets, defined by $\left[Q_{\xi}, Q_{\zeta}\right]=-\delta_{\xi} Q_{\zeta}$, read

$$
\begin{aligned}
i\left[T_{n}, T_{m}\right] & =(n-m) T_{n+m}, \\
i\left[T_{n}, P_{m}\right] & =-m P_{n+m}-i \kappa n^{2} \delta_{n+m, 0}, \\
i\left[P_{n}, P_{m}\right] & =0,
\end{aligned}
$$

\footnotetext{
${ }^{5}$ Note that the change of variables (3.10) is invertible for functions on the circle: given the functions $a$ and $\eta$, eq. (3.10) specifies $T$ and $P$ uniquely; conversely, given some functions $T$ and $P$, the functions $a$ and $\eta$ ensuring that (3.10) holds are unique provided one imposes $2 \pi L$-periodicity of the coordinate $u$.
} 
with $\kappa=k$. As it must be for a consistent theory, this algebra coincides with the Lie bracket algebra (2.25) of allowed diffeomorphisms, up to central extensions. Note that $T_{0} / L$, being the charge that generates time translations $u \mapsto u+$ const, should be interpreted as the Hamiltonian, while $P_{0}$ is the momentum operator (it generates translations $x \mapsto x+$ const). The only central extension in (3.14) is a twist term in the mixed commutator; it is a nontrivial 2-cocycle [104]. In particular, it cannot be removed by redefinitions of generators since the $u(1)$ current algebra has a vanishing level.

\subsection{Warped Virasoro group and coadjoint representation}

As a preliminary step towards the computation of quasi-Rindler entropy, we now work out the finite transformation laws of the functions $T$ and $P$. For the sake of generality, we will display the result for arbitrary central extensions of the warped Virasoro group, including a Virasoro central charge and a $u(1)$ level. We will use a $2 \pi L$-periodic coordinate $u$, but our end result (3.17)-(3.18) actually holds independently of that assumption.

Finite transformations of the stress tensor. The asymptotic symmetry group for quasi-Rindler holography is (3.3) with $\mathbb{R}$ replaced by $S^{1}$, and it consists of pairs $(f, p)$, where $p$ is an arbitrary function on the circle and $f$ is a diffeomorphism of the circle; in particular,

$$
f(u+2 \pi L)=f(u)+2 \pi L .
$$

(For instance, the diffeomorphisms defined by (3.7) are generally forbidden once that condition is imposed.) However, in order to accommodate for inhomogeneous terms such as those appearing in the infinitesimal transformations (2.30), we actually need to study the central extension of this group. We will call this central extension the warped Virasoro group, and we will denote it by $\hat{G}$. Its Lie algebra reads

$$
\begin{aligned}
i\left[T_{n}, T_{m}\right] & =(n-m) T_{n+m}+\frac{c}{12} n^{3} \delta_{n+m, 0} \\
i\left[T_{n}, P_{m}\right] & =-m P_{n+m}-i \kappa n^{2} \delta_{n+m, 0} \\
i\left[P_{n}, P_{m}\right] & =K n \delta_{n+m, 0}
\end{aligned}
$$

and is thus an extension of (3.14) with a Virasoro central charge $c$ and a $u(1)$ level $K$. Note that, when $K \neq 0$, the central term in the mixed commutator $[T, P]$ can be removed by defining $\mathcal{L}_{n} \equiv T_{n}+\frac{i \kappa}{K} n P_{n}$. In terms of generators $\mathcal{L}_{n}$ and $P_{n}$, the algebra takes the form (3.16) without central term in the mixed bracket, and with a new Virasoro central charge $c^{\prime}=c-12 \kappa^{2} / K$. [We will see an illustration of this in eq. (5.11), in the context of quasi-Rindler gravity in $\mathrm{AdS}_{3}$.] But when $K=0$ as in (3.14), there is no such redefinition.

We relegate to appendix A.1 the exact definition of the warped Virasoro group $\hat{G}$, together with computations related to its adjoint and coadjoint representations. Here we simply state the result that is important for us, namely the finite transformation law of the stress tensor $T$ and the $u(1)$ current $P$. By construction, these transformations coincide with the coadjoint representation of $\hat{G}$, written in eqs. (A.21)-(A.22); thus, under a finite 
transformation $(3.2)$, the pair $(T, P)$ is mapped to a new pair $(\tilde{T}, \tilde{P})$ with

$$
\begin{array}{r}
\tilde{T}(f(u))=\frac{1}{\left(f^{\prime}(u)\right)^{2}}\left[T(u)+\frac{c}{12 k}\{f ; u\}-P(u)(p \circ f)^{\prime}(u)\right. \\
\left.-\frac{\kappa}{k}(p \circ f)^{\prime \prime}(u)+\frac{K}{2 k}\left((p \circ f)^{\prime}(u)\right)^{2}\right] \\
\tilde{P}(f(u))=\frac{1}{f^{\prime}(u)}\left[P(u)+\frac{\kappa}{k} \frac{f^{\prime \prime}(u)}{f^{\prime}(u)}-\frac{K}{k}(p \circ f)^{\prime}(u)\right],
\end{array}
$$

where $\{f ; u\}$ is the Schwarzian derivative (A.10) of $f$. These transformations extend those of a standard warped CFT [86], which are recovered for $\kappa=0$. In the case of quasi-Rindler spacetimes, we have $c=K=0$ and $\kappa$ is non-zero, leading to

$$
\begin{aligned}
& \tilde{T}(f(u))=\frac{1}{\left(f^{\prime}(u)\right)^{2}}\left[T(u)-P(u)(p \circ f)^{\prime}(u)-\frac{\kappa}{k}(p \circ f)^{\prime \prime}(u)\right] \\
& \tilde{P}(f(u))=\frac{1}{f^{\prime}(u)}\left[P(u)+\frac{\kappa}{k} \frac{f^{\prime \prime}(u)}{f^{\prime}(u)}\right]
\end{aligned}
$$

which (for $\kappa=k$ ) actually follows from (3.6) and the definition (3.10). Note that these formulas are valid regardless of whether $u$ is periodic or not! In the latter case, $f(u)$ is a diffeomorphism of the real line.

Modified Sugawara construction. Before going further, we note the following: since $P(u)$ is a Kac-Moody current, one expects that a (possibly modified) Sugawara construction might convert some quadratic combination of $P$ 's into a CFT stress tensor. This expectation is compatible with the fact that $P(u) \mathrm{d} u$ is a one-form, so that $P(u) \mathrm{d} u \otimes P(u) \mathrm{d} u$ is a quadratic density. Let us therefore define

$$
\mathcal{M}(u) \equiv \frac{k^{2}}{2 \kappa}(P(u))^{2}+k P^{\prime}(u)
$$

and ask how it transforms under the action of $(f, p)$, given that the transformation law of $P(u)$ is (3.20). Writing this transformation as $\mathcal{M} \longmapsto \widetilde{\mathcal{M}}$, the result is

$$
\widetilde{\mathcal{M}}(f(u))=\frac{1}{\left(f^{\prime}(u)\right)^{2}}[\mathcal{M}(u)+\kappa\{f ; u\}]
$$

which is the transformation law of a CFT stress tensor with central charge

$$
c_{M} \equiv 12 \kappa=12 k=\frac{3}{G_{N}} .
$$

Once more, this observation is independent of whether the coordinate $u$ is periodic or not.

This construction is at the core of a simple relation between the quasi-Rindler symmetry algebra (3.14) and the $\mathrm{BMS}_{3}$ algebra. Indeed, by quadratically recombining the generators $P_{n}$ thanks to a twisted Sugawara construction

$$
M_{n}=\frac{1}{2 \kappa} \sum_{q \in \mathbb{Z}} P_{n-q} P_{q}-i n P_{n},
$$


the brackets (3.14) reproduce the centrally extended $\mathrm{BMS}_{3}$ algebra [14]:

$$
\begin{aligned}
i\left[T_{n}, T_{m}\right] & =(n-m) T_{n+m} \\
i\left[T_{n}, M_{m}\right] & =(n-m) M_{n+m}+\frac{c_{M}}{12} n^{3} \delta_{n+m, 0} \\
i\left[M_{n}, M_{m}\right] & =0 .
\end{aligned}
$$

This apparent coincidence implies that the representations of the warped Virasoro group (with vanishing Kac-Moody level) are related to those of the $\mathrm{BMS}_{3}$ group, but one should keep in mind that the similarity of group structures does not imply similarity of the physics involved; in particular, the Hamiltonian operator in (3.25) is (proportional to) $T_{0}$, while the standard $\mathrm{BMS}_{3}$ Hamiltonian is $M_{0}$. Nevertheless, unitary representations of the warped Virasoro group with vanishing Kac-Moody level $K$ can indeed be studied and classified along the same lines as for $\mathrm{BMS}_{3}[26,27]$. As we do not use these results in the present work we relegate this discussion to appendix A.2.

\section{Quasi-Rindler thermodynamics}

In this section we study quasi-Rindler thermodynamics, both microscopically and macroscopically, assuming throughout that surface charges are defined as integrals over time and that the coordinate $u$ is $2 \pi L$-periodic. We start in subsection 4.1 with a microscopic, Cardy-inspired derivation of the entropy of zero-mode solutions. Section 4.2 is devoted to certain geometric aspects of boosted Rindler spacetimes, for instance their global Killing vectors, which has consequences for our analytic continuation to Euclidean signature in subsection 4.3. In subsection 4.4 we evaluate the on-shell action to determine the free energy, from which we then derive other thermodynamic quantities of interest, such as macroscopic quasi-Rindler entropy. In particular, we exhibit the matching between the Cardy-based computation and the purely gravitational one.

\subsection{Modular invariance and microscopic entropy}

Here, following [86], we switch on chemical potentials (temperature and velocity) and compute the partition function in the high-temperature limit, assuming the validity of a suitable version of modular invariance. Because the $u(1)$ level vanishes in the present case, the modular transformations will not be anomalous, in contrast to standard behaviour in warped CFT [86]. (This will no longer be true in $\mathrm{AdS}_{3}$ — see section 5.)

The grand canonical partition function of a theory at temperature $1 / \beta$ and velocity $\eta$ is

$$
Z(\beta, \eta)=\operatorname{Tr}\left(e^{-\beta(H-\eta P)}\right),
$$

where $H$ and $P$ are the Hamiltonian and momentum operators (respectively), and the trace is taken over the Hilbert space of the system. In the present case, the Hamiltonian is the (quantization of the) charge (3.11) associated with $t(u)=1$ and $p(u)=0$, i.e. the zero-mode of $T(u)$ up to normalization:

$$
H=\frac{k}{2 \pi} \int_{0}^{2 \pi L} \mathrm{~d} u T(u) .
$$


As for the momentum operator, it is the zero-mode of $P(u)$ (again, up to normalization). If we denote by $I$ the Euclidean action of the system, the partition function (4.1) can be computed as the integral of $e^{-I+\eta \int \mathrm{d} \tau P}$ over paths in phase space that are periodic in Euclidean time $\tau$ with period $\beta$. Equivalently, if we assume that the phase space contains one Lagrange variable at each point of space (i.e. that we are dealing with a field theory), the partition function may be seen as a path integral of $e^{-I}$ over fields $\phi$ that satisfy $\phi(\tau+\beta, x)=\phi(\tau, x+i \beta \eta)$ since $P$ is the generator of translations along $x$. Note that both approaches require the combination $H-\eta P$ to be bounded from below; in typical cases (such as AdS, where $P$ is really an angular momentum operator and $\eta$ is an angular velocity), this is a restriction on the allowed velocities of the system.

Now, our goal is to find an asymptotic expression for the partition function at high temperature. To do this, we will devise a notion of modular invariance (actually only $S$-invariance), recalling that the symmetries of our theory are transformations of (the complexification of) $S^{1} \times \mathbb{R}$ of the form (3.2). Seeing the partition function (4.1) as a path integral, the variables that are integrated out live on a plane $\mathbb{R}^{2}$ spanned by coordinates $u$ and $x$ subject to the identifications

$$
(u, x) \sim(u+i \beta, x-i \beta \eta) \sim(u+2 \pi L, x) .
$$

The transformations

$$
\tilde{u}=\frac{2 \pi i L}{\beta} u \quad \tilde{x}=x+\eta u
$$

map these identifications on

$$
(\tilde{u}, \tilde{x}) \sim(\tilde{u}-2 \pi L, \tilde{x}) \sim\left(\tilde{u}+i \frac{(2 \pi L)^{2}}{\beta}, \tilde{x}+2 \pi L \eta\right) .
$$

While the transformations (4.4) do not belong to the group of finite asymptotic symmetry transformations (3.2), their analogues in the case of CFT's, $\mathrm{BMS}_{3}$-invariant theories and warped CFT's $[20,21,86]$ apparently lead to the correct entropy formulas. We shall assume that the same is true here, which implies that the partition function $Z(\beta, \eta)$ satisfies a property analogous to self-reciprocity,

$$
Z(\beta, \eta)=Z\left(\frac{(2 \pi L)^{2}}{\beta}, \frac{i \beta \eta}{2 \pi L}\right)
$$

This, in turn, gives the asymptotic formula

$$
Z(\beta, \eta) \stackrel{\beta \rightarrow 0^{+}}{\sim} \exp \left[-\frac{(2 \pi L)^{2}}{\beta}\left(H_{\mathrm{vac}}-\frac{i \beta \eta}{2 \pi L} P_{\mathrm{vac}}\right)\right],
$$

where $H_{\text {vac }}$ and $P_{\text {vac }}$ are respectively the energy and momentum of the vacuum state. These values can be obtained from the finite transformations (3.19) [with $T=P=p=0$ ] and (3.20) [with $P=0$ ] by considering the map $f(u)=L e^{i n u / L}$ with some integer $n$ and declaring that the vacuum value of the functions $T(u)$ and $P(u)$ is zero, exactly like for the map between the plane and the cylinder in a CFT. Accordingly, the vacuum values of these functions "on the cylinder", say $\tilde{T}_{\text {vac }}$ and $\tilde{P}_{\text {vac }}$, are

$$
\tilde{T}_{\text {vac }}=0 \quad f^{\prime}(u) \tilde{P}_{\text {vac }}=\frac{i n}{L} \cdot \frac{\kappa}{k},
$$


so that $H_{\mathrm{vac}}=0$. Choosing $|n| \neq 1$ introduces a conical excess (see subsection 4.3), or equivalently gives a map $u \mapsto L e^{i n u / L}$ which is not injective, so the only possible choices are $n= \pm 1$. Using $P_{\text {vac }}=\frac{k}{2 \pi} \int \mathrm{d} f \tilde{P}_{\text {vac }}(f(u))=\frac{k}{2 \pi} \int_{0}^{2 \pi L} \mathrm{~d} u f^{\prime} \tilde{P}_{\text {vac }}$ then establishes

$$
P_{\mathrm{vac}}= \pm i \kappa \quad \text { for } n= \pm 1 \text {. }
$$

The asymptotic expression (4.7) of the partition function thus becomes

$$
Z(\beta, \eta) \stackrel{\beta \rightarrow 0^{+}}{\sim} e^{2 \pi L \kappa|\eta|},
$$

where the sign of the dominant vacuum value in (4.9) is determined by the sign of $\eta$. (More precisely, the vacuum $\pm i \kappa$ is selected when $\operatorname{sign}(\eta)=\mp 1$.) The free energy $F \equiv-T \log Z$ is given by

$$
F \approx-2 \pi \kappa L|\eta| T
$$

at high temperature, and the corresponding entropy is

$$
S=-\left.\frac{\partial F}{\partial T}\right|_{\eta} \approx 2 \pi \kappa L|\eta|=2 \pi k L|\eta|=\frac{2 \pi L|\eta|}{4 G_{N}} .
$$

In subsection 4.4 we will see that this result exactly matches that of a macroscopic (i.e. purely gravitational) computation. Before doing so, we study Euclidean quasi-Rindler spacetimes and elucidate the origin of the vacuum configuration (4.8).

\subsection{Boosted Rindler spacetimes and their Killing vectors}

Any solution of 3-dimensional Einstein gravity is locally flat and therefore locally has six Killing vector fields. However, these vector fields may not exist globally. We now discuss global properties of the Killing vectors of the geometry defined by the line-element (2.1) with the identification (2.23). For simplicity, we present our results only for zero-mode solutions, $a, \eta=$ const.

The six local Killing vector fields are

$$
\begin{array}{ll}
\xi_{1}=\partial_{u} & \xi_{4}=e^{a u}\left(\partial_{u}-\eta \partial_{x}-\left(a r+\frac{1}{2} \eta^{2}\right) \partial_{r}\right) \\
\xi_{2}=\partial_{x} & \xi_{5}=a(\eta u+x) \xi_{3}-e^{-a u} \partial_{x} \\
\xi_{3}=e^{-a u} \partial_{r} & \xi_{6}=a(\eta u+x) \xi_{4}+e^{a u}\left(a r+\frac{1}{2} \eta^{2}\right) \partial_{x} .
\end{array}
$$

Globally, due to our identification (2.23), only the Killing vectors $\xi_{1}$ and $\xi_{2}$ survive for generic values of $a$. The only exception arises for specific imaginary values of Rindler acceleration,

$$
a=\frac{i n}{L} \quad 0 \neq n \in \mathbb{Z},
$$

in which case $\xi_{3}$ and $\xi_{4}$ are globally well-defined as well. If in addition $\eta=0$, then all six Killing vector fields can be defined globally. The non-vanishing Lie brackets between the 
Killing vectors are

$$
\begin{array}{llrl}
{\left[\xi_{1}, \xi_{3}\right]} & =-\left[\xi_{2}, \xi_{5}\right]=-a \xi_{3} & {\left[\xi_{1}, \xi_{5}\right]=a \eta \xi_{3}-a \xi_{5}} \\
{\left[\xi_{1}, \xi_{4}\right]=\left[\xi_{2}, \xi_{6}\right]=a \xi_{4}} & {\left[\xi_{1}, \xi_{6}\right]=a \eta \xi_{4}+a \xi_{6}} \\
{\left[\xi_{3}, \xi_{6}\right]=\left[\xi_{4}, \xi_{5}\right]=a \xi_{2}} & {\left[\xi_{5}, \xi_{6}\right]=a \eta \xi_{2}-a \xi_{1} .}
\end{array}
$$

This algebra is isomorphic to $i s l(2)$, as displayed in (2.4), with the identifications $M_{0}=\xi_{2}$, $M_{+}=-2 \xi_{4}, M_{-}=-\xi_{3}, L_{0}=\left(-\xi_{1}+\eta \xi_{2}\right) / a, L_{+}=2\left(\xi_{6}+\eta \xi_{4}\right) / a, L_{-}=-\left(\xi_{5}+\eta \xi_{3}\right) / a$. In terms of the generators $t_{n}, p_{n}$ of the asymptotic Lie bracket algebra (2.25) we have the identifications $t_{0} \sim \xi_{1}, p_{0} \sim \xi_{2}, t_{1} \sim \xi_{4}$ and $p_{-1} \sim \xi_{5}$; the vector field $\xi_{3}$ generates trivial symmetries, while the Killing vector $\xi_{6}$ is not an asymptotic Killing vector, as it is incompatible with the asymptotic behavior (2.21). This shows in particular that the boundary conditions of quasi-Rindler gravity actually break Poincaré symmetry even when the coordinate $u$ is not periodic. Interestingly, the four generators $t_{0}, t_{1}, p_{-1}, p_{0}$ obey the harmonic oscillator algebra

$$
i\left[\alpha, \alpha^{\dagger}\right]=z \quad i[H, \alpha]=-\alpha \quad i\left[H, \alpha^{\dagger}\right]=\alpha^{\dagger}
$$

where the Hamiltonian is formally given by $t_{0}=H=\alpha^{\dagger} \alpha+$ const., the annihilation/creation operators formally by $t_{1}=\alpha, p_{-1}=\alpha^{\dagger}$ and $p_{0}=z$ commutes with the other three generators. The algebra is written here in terms of Poisson brackets, but becomes the standard harmonic oscillator algebra after quantization. Note, however, that $t_{1}$ and $p_{-1}$ are not generally adjoint to each other. ${ }^{6}$ In the canonical realization (3.14) the first commutator acquires an important contribution from the central extension

$$
i\left[T_{1}, P_{-1}\right]=P_{0}-i \kappa .
$$

If we wish to identify the vacuum as the most symmetric solution then our vacuum spacetime takes the form

$$
\mathrm{d} s^{2}=-\frac{2 i n r}{L} \mathrm{~d} u^{2}-2 \mathrm{~d} u \mathrm{~d} r+\mathrm{d} x^{2}
$$

with some non-zero integer $n$. We shall demonstrate in subsection 4.3 that $|n|=1$ is the only choice consistent with the $u$-periodicity (2.23). Thus, we have uncovered yet another unusual feature of quasi-Rindler holography: the vacuum metric (4.18) with $|n|=1$ is complex. Our vacuum is neither flat spacetime (as one might have guessed naively) nor a specific Rindler spacetime, but instead it is a Rindler-like spacetime with a specific imaginary Rindler "acceleration", the value of which depends on the choice of the periodicity $L$ in (2.23). Another way to see that the solution $a=\eta=0$ is not maximally symmetric for finite $L$ is to consider the six local Killing vectors $\xi_{i}^{(0)}$ in the limit $a, \eta \rightarrow 0$ :

$$
\begin{array}{ll}
\xi_{1}^{(0)}=\partial_{u} & \xi_{4}^{(0)}=u \partial_{u}-r \partial_{r} \\
\xi_{2}^{(0)}=\partial_{x} & \xi_{5}^{(0)}=u \partial_{x}+x \partial_{r} \\
\xi_{3}^{(0)}=\partial_{r} & \xi_{6}^{(0)}=x \partial_{u}+r \partial_{x}
\end{array}
$$

\footnotetext{
${ }^{6}$ More precisely, they are certainly not each other's adjoint in a unitary representation, although we seem to be dealing with a non-unitary representation anyway (the vacuum value of $P_{0}$ is imaginary).
} 
Only four of them can be defined globally, because $\xi_{4}^{(0)}$ and $\xi_{5}^{(0)}$ have a linear dependence on $u$ that is incompatible with finite $2 \pi L$-periodicity (2.23).

While the vacuum metric (4.18) (with $n=1$ ) is preserved by all six Killing vectors $(4.13 \mathrm{c}$ ), at most three of the associated generators of the asymptotic symmetry algebra can annihilate the vacuum; since we expect from the discussion in subsection 4.1 that $P_{0}$ is non-zero we pick the vacuum by demanding that $T_{0}, T_{1}$ and $P_{-1}$ annihilate it. The generator $P_{0}$ then acquires a non-zero eigenvalue due to the central term in (4.17),

$$
T_{0}|0\rangle=T_{1}|0\rangle=P_{-1}|0\rangle=\left[T_{1}, P_{-1}\right]|0\rangle=0 \quad \Rightarrow \quad P_{0}|0\rangle=i \kappa|0\rangle=\frac{i}{4 G_{N}}|0\rangle
$$

while the remaining two Killing vectors are excluded for reasons stated above (one acts trivially, while the other violates the boundary conditions for asymptotic Killing vectors). In fact, upon performing a shift $P_{0} \rightarrow P_{0}-i \kappa$, the symmetry algebra (3.14) becomes

$$
\begin{aligned}
& i\left[T_{n}, T_{m}\right]=(n-m) T_{n+m}, \\
& i\left[T_{n}, P_{m}\right]=-m P_{n+m}-i \kappa n(n-1) \delta_{n+m, 0}, \\
& i\left[P_{n}, P_{m}\right]=0
\end{aligned}
$$

so that the vacuum is now manifestly invariant under $T_{0}, T_{1}, P_{0}$ and $P_{-1}$.

These considerations reproduce the result (4.9) and thereby provide a consistency check. The fact that the eigenvalue of $P_{0}$ is imaginary indicates that the warped Virasoro group is represented in a non-unitary way if the assumption of self-reciprocity (4.6) holds.

\subsection{Euclidean boosted Rindler}

In order to prepare the ground for thermodynamics, we now study the Euclidean version of the metrics (2.1). We consider only zero-mode solutions for simplicity. Then, defining new coordinates

$$
\tau=u+\frac{1}{2 a} \ln \left(2 a r+\eta^{2}\right) \quad y=x-\frac{\eta}{2 a} \ln \left(2 a r+\eta^{2}\right) \quad \rho=r+\frac{\eta^{2}}{2 a},
$$

the line-element (2.1) becomes

$$
-2 a \rho \mathrm{d} \tau^{2}+\frac{\mathrm{d} \rho^{2}}{2 a \rho}+(\mathrm{d} y+\eta \mathrm{d} \tau)^{2} .
$$

For non-zero Rindler acceleration, $a \neq 0$, there is a Killing horizon at $\rho_{h}=0$, or equivalently at

$$
r=r_{h}=-\frac{\eta^{2}}{2 a} .
$$

The patch $\rho>0$ coincides with the usual Rindler patch for positive Rindler acceleration $a$. For negative $a$ the patches $\rho>0$ and $\rho<0$ switch their roles. We assume positive $a$ in this work so that $\tau$ is a timelike coordinate in the limit $r \rightarrow \infty$.

The Euclidean section of the metric (4.23) is obtained by defining

$$
t_{\mathrm{E}}=-i \tau
$$


which yields the line-element

$$
\mathrm{d} s^{2}=2 a \rho \mathrm{d} t_{\mathrm{E}}^{2}+\frac{\mathrm{d} \rho^{2}}{2 a \rho}+\left(\mathrm{d} y+i \eta \mathrm{d} t_{\mathrm{E}}\right)^{2} .
$$

Demanding the absence of a conical singularity at $\rho=0$ and compatibility with (2.23) leads to the periodicities

$$
\left(t_{\mathrm{E}}, y\right) \sim\left(t_{\mathrm{E}}+\beta, y-i \beta \eta\right) \sim\left(t_{\mathrm{E}}-2 \pi i L, y\right) .
$$

which are the Euclidean version of the periodicities (4.3), with the inverse temperature $\beta=T^{-1}$ given by

$$
T=\frac{a}{2 \pi} .
$$

Given the periodicities (4.27), we can now ask which values of $n$ in (4.14) give rise to a regular spacetime with metric (4.18). Consider the Euclidean line-element (4.26) and define another analytic continuation,

$$
a=\frac{i n}{L} \quad \tau=i t_{\mathrm{E}} \quad \hat{\rho}=-i \rho \operatorname{sign}(n),
$$

which yields

$$
\mathrm{d} s^{2}=\frac{2|n| \hat{\rho} \mathrm{d} \tau^{2}}{L}+\frac{L \mathrm{~d} \hat{\rho}^{2}}{2|n| \hat{\rho}}+(\mathrm{d} y+\eta \mathrm{d} \tau)^{2} .
$$

with the periodicities

$$
(\tau, y) \sim(\tau+i \beta, y-i \beta \eta) \sim(\tau+2 \pi L, y) .
$$

The point now is that the Euclidean line-element (4.30) with the periodicities (4.31) has a conical singularity at $\hat{\rho}=0$ unless $|n|=1$. Thus, we conclude that the vacuum spacetime (in the sense of being singularity-free and maximally symmetric) is given by (4.18) with $|n|=1$, confirming our discussion in sections 4.1 and 4.2 .

\subsection{Macroscopic free energy and entropy}

The saddle point approximation of the Euclidean path integral leads to the Euclidean partition function, which in turn yields the free energy. The latter is given by temperature times the Euclidean action (2.20) evaluated on-shell:

$$
F=-\left.T \frac{1}{8 \pi G_{N}} \int_{0}^{-i 2 \pi L} \mathrm{~d} t_{\mathrm{E}} \int_{0}^{i \beta|\eta|} \mathrm{d} y \sqrt{\gamma} K\right|_{\rho \rightarrow \infty}=-\frac{2 \pi L|\eta| T}{4 G_{N}}
$$

where we have inserted the periodicities from subsection 4.3 and used $\sqrt{\gamma} K=a+\mathcal{O}(1 / \rho)$. The absolute value for $\eta$ was introduced in order to ensure a positive volume form ${ }^{7}$ for positive $L$ and $\beta$.

\footnotetext{
${ }^{7}$ One pragmatic way to get the correct factors of $i$ is to insert the Euclidean periodicities in the ranges of the integrals and to demand again positive volume when integrating the function 1 . In the flat space calculation this implies integrating $u$ from 0 to $\beta$, while here it implies integrating $u$ from 0 to $-i 2 \pi L$.
} 
From this result we extract the entropy

$$
S=-\left.\frac{\partial F}{\partial T}\right|_{\eta}=\frac{2 \pi L|\eta|}{4 G_{N}}
$$

which coincides with the Cardy-based result (4.12). As a cross-check, we derive the same expression in the Chern-Simons formulation by analogy to the flat space results $[44,45]$ :

$$
S=\frac{k}{2 \pi} \int_{0}^{-i 2 \pi L} \mathrm{~d} u \int_{0}^{i \beta|\eta|} \mathrm{d} x\left\langle A_{u} A_{x}\right\rangle=k L \beta|\eta|\left\langle\mathfrak{a}_{u} \mathfrak{a}_{x}\right\rangle=2 \pi k L|\eta|=\frac{2 \pi L|\eta|}{4 G_{N}} .
$$

We show in the next section that the same matching occurs in Rindler-AdS.

\section{Boosted Rindler-AdS}

In this section we generalize the discussion of the previous pages to the case of RindlerAdS spacetimes. In subsection 5.1 we establish quasi-Rindler-AdS boundary conditions and show that the asymptotic symmetry algebra can be untwisted to yield a standard warped CFT algebra, with a $u(1)$ level that vanishes in the limit of infinite AdS radius. Then, in subsection 5.2 we derive the entropy microscopically, and we show in subsection 5.3 that the same result can be obtained macroscopically.

\subsection{Boundary conditions and symmetry algebra}

We can deform the metric (2.1) to obtain a solution of Einstein's equations $R_{\mu \nu}-\frac{1}{2} g_{\mu \nu} R=$ $g_{\mu \nu} / \ell^{2}$ with a negative cosmological constant $\Lambda=-1 / \ell^{2}$,

$$
\mathrm{d} s^{2}=-2 a(u) r \mathrm{~d} u^{2}-2 \mathrm{~d} u \mathrm{~d} r+2(\eta(u)+2 r / \ell) \mathrm{d} u \mathrm{~d} x+\mathrm{d} x^{2} .
$$

Starting from this ansatz, we now adapt our earlier discussion to the case of a non-vanishing cosmological constant. Since the computations are very similar to those of the quasi-Rindler case, we will simply point out the changes that arise due to the finite AdS radius. When we do not mention a result explicitly we imply that it is the same as for the flat configuration; in particular we assume again $2 \pi L$-periodicity in $u$.

The Chern-Simons formulation is based on the deformation of the $i s l(2)$ algebra to $s o(2,2)$, where the translation generators no longer commute so that the last bracket in $(2.4)$ is replaced by

$$
\left[M_{n}, M_{m}\right]=\frac{1}{\ell^{2}}(n-m) L_{n+m}
$$

The on-shell connection (2.10)-(2.12) and the asymptotic symmetry generators (2.27)(2.29) are modified as

$$
\mathfrak{a} \rightarrow \mathfrak{a}+\Delta \mathfrak{a} / \ell, \quad \varepsilon \rightarrow \varepsilon+\Delta \varepsilon / \ell \quad \text { and } \quad b=\exp \left[\frac{r}{2}\left(M_{-1}-\frac{1}{\ell} L_{-1}\right)\right]
$$


where

$$
\begin{aligned}
& \Delta \mathfrak{a}=\mathrm{d} u L_{1}-\mathrm{d} x L_{0}+\frac{1}{2} \eta(u) \mathrm{d} x L_{-1} \\
& \Delta \varepsilon=t(u) L_{1}-p(u) L_{0}-\Upsilon(u) L_{-1} .
\end{aligned}
$$

The connection $A$ changes correspondingly as compared to (2.13),

$$
A \rightarrow A+\Delta A / \ell \quad \Delta A=\Delta \mathfrak{a}-\frac{\mathrm{d} r}{2} L_{-1}+r\left(\frac{1}{\ell} L_{-1} \mathrm{~d} x-M_{-1} \mathrm{~d} x-\frac{1}{2} a(u) L_{-1} \mathrm{~d} u\right) .
$$

Note in particular that all quadratic terms in $r$ cancel due to the identity $\left[L_{-1},\left[L_{-1}, \mathfrak{a}\right]\right]-$ $2 \ell\left[L_{-1},\left[M_{-1}, \mathfrak{a}\right]\right]+\ell^{2}\left[M_{-1},\left[M_{-1}, \mathfrak{a}\right]\right]=0$. Plugging the result (5.5) into the lineelement (2.6) yields

$$
\mathrm{d} s^{2} \rightarrow \mathrm{d} s^{2}+\Delta \mathrm{d} s^{2} / \ell \quad \Delta \mathrm{d} s^{2}=4 r \mathrm{~d} u \mathrm{~d} x
$$

thus reproducing the solution (5.1).

Consequently, the variations of the functions $a(u)$ and $\eta(u)$ in (2.30) are also modified,

$$
\begin{aligned}
& \delta a(u) \rightarrow \delta a(u)-2 p^{\prime}(u) / \ell, \\
& \delta \eta(u) \rightarrow \delta \eta(u)+2 p(u) \eta(u) / \ell+4 \Upsilon(u) / \ell .
\end{aligned}
$$

Using (2.29), one can show that the presence of the last term in the second line does not affect the transformation of the function $T(u)$ defined in (3.10). Moreover, the charges (3.11) remain unchanged. In fact, in the Rindler-AdS case only the transformation of the current $P$ is deformed as

$$
\delta_{p} P=-2 p^{\prime} / \ell
$$

which leads to the following Poisson brackets of the charges $P_{n}$ defined in (3.13):

$$
i\left[P_{n}, P_{m}\right]=-\frac{2 k}{\ell} n \delta_{n+m, 0} .
$$

In particular, the limit $\ell \rightarrow \infty$ reproduces the algebra (3.14).

At finite $\ell$ the presence of the non-vanishing level in (5.10) enables us to remove the central extension of the mixed bracket of $(3.14 \mathrm{~b})$ thanks to a twist

$$
\mathcal{L}_{n}=T_{n}-\frac{i \ell \kappa}{2 k} n P_{n}
$$

in terms of which the asymptotic symmetry algebra reads

$$
\begin{aligned}
i\left[\mathcal{L}_{n}, \mathcal{L}_{m}\right] & =(n-m) \mathcal{L}_{n+m}+\frac{c}{12} n^{3} \delta_{n+m, 0} \\
i\left[\mathcal{L}_{n}, P_{m}\right] & =-m P_{n+m} \\
i\left[P_{n}, P_{m}\right] & =-\frac{2 k}{\ell} n \delta_{n+m, 0}
\end{aligned}
$$

with the expected Brown-Henneaux central charge ${ }^{8}[8]$

$$
c=6 \frac{\kappa^{2}}{k} \ell=6 k \ell=\frac{3 \ell}{2 G_{N}} .
$$

\footnotetext{
${ }^{8}$ This central charge is expected to be shifted quantum mechanically at finite $k \ell$ [92, 93]. Since we are interested in the semi-classical limit here, we shall not take such a shift into account.
} 


\subsection{Microscopic quasi-Rindler-AdS entropy}

As in the case of a vanishing cosmological constant, it is possible to derive a Cardy-like entropy formula that can be applied to zero-mode solutions. The only difference with respect to subsection 4.1 is the non-vanishing $u(1)$ level $K=-2 k / \ell$ that leads to a slightly different form of modular invariance. Namely, according to [86], the self-reciprocity of the partition function, eq. (4.6), now becomes

$$
Z(\beta, \hat{\eta})=e^{\beta K L \hat{\eta}^{2} / 2} Z\left(\frac{(2 \pi L)^{2}}{\beta}, \frac{i \beta \hat{\eta}}{2 \pi L}\right)
$$

leading to the high-temperature free energy

$$
F \approx \frac{(2 \pi L)^{2}}{\beta^{2}}\left(H_{\mathrm{vac}}-\frac{i \beta \hat{\eta}}{2 \pi L} P_{\mathrm{vac}}\right)-K L \hat{\eta}^{2} / 2
$$

(We have renamed the chemical potential conjugate to $P_{0}$ as $\hat{\eta}$ for reasons that will become clear in subsection 5.3.) This is the same as in (4.7), up to a temperature-independent constant proportional to the $u(1)$ level. The vacuum values of the Hamiltonian and the momentum operator are once more given by the arguments above (4.8); in particular, the $u(1)$ level plays no role for these values. Accordingly, the free energy at high temperature $T=\beta^{-1} \gg 1$ boils down to

$$
F \approx-2 \pi L k|\hat{\eta}| T+k L \hat{\eta}^{2} / \ell
$$

and the corresponding entropy is again given by (4.12):

$$
S=\frac{2 \pi L|\hat{\eta}|}{4 G_{N}}
$$

Notably, this is independent of the AdS radius. The same result can be obtained by absorbing the twist central charge through the redefinition (5.11) and then using the Cardy-like entropy formula derived in [86]. In the next subsection we show that this result coincides with the gravitational entropy, as in the flat quasi-Rindler case discussed previously.

\subsection{Macroscopic quasi-Rindler-AdS entropy}

Generalizing the macroscopic calculations from section 4 for zero-mode solutions (5.3) with constant $a$ and $\eta$ we find that the outermost Killing horizon is located at

$$
r_{h}=\frac{\ell}{4}\left(\sqrt{a^{2} \ell^{2}+4 a \ell \eta}-a \ell-2 \eta\right)=-\frac{\eta^{2}}{2 a}+\mathcal{O}(1 / \ell)
$$

and has a smooth limit to the quasi-Rindler result (4.24) for infinite AdS radius $\ell \rightarrow \infty$. We assume $\eta>-a \ell / 4$ so that $r_{h}$ is real and surface-gravity is non-zero.

The vacuum spacetime reads

$$
\mathrm{d} s^{2}=-\frac{2 i r \mathrm{~d} u^{2}}{L}-2 \mathrm{~d} u \mathrm{~d} r+\frac{4 r}{\ell} \mathrm{d} u \mathrm{~d} x+\mathrm{d} x^{2}
$$


where again we defined "vacuum" as the unique spacetime compatible with our boundary conditions, regularity and maximal symmetry.

Making a similar analytic continuation as in subsection 4.3 we obtain the line-element

$$
\mathrm{d} s^{2}=K(r) \mathrm{d} t_{\mathrm{E}}^{2}+\frac{\mathrm{d} r^{2}}{K(r)}+\left(\mathrm{d} y+i(2 r / \ell+\eta) \mathrm{d} t_{\mathrm{E}}\right)^{2}
$$

with the Killing norm

$$
K(r)=\frac{4 r^{2}}{\ell^{2}}+\frac{4 \eta r}{\ell}+2 a r+\eta^{2}
$$

and the periodic identifications

$$
\left(t_{\mathrm{E}}, y\right) \sim\left(t_{\mathrm{E}}-2 \pi i L, y\right) \sim\left(t_{\mathrm{E}}+\beta, y-i \beta \hat{\eta}\right)
$$

where inverse temperature $\beta=T^{-1}$ and boost parameter $\hat{\eta}$ are given by

$$
T=\frac{\sqrt{a^{2}+4 a \eta / \ell}}{2 \pi} \quad \hat{\eta}=\sqrt{a^{2} \ell^{2} / 4+a \eta \ell}-a \ell / 2 .
$$

In particular, the chemical potential $\hat{\eta}$ no longer coincides with the parameter $\eta$ appearing in the metric. Note that the limit of infinite AdS radius is smooth and leads to the expressions in subsection 4.3 for line-element, periodicities, temperature and boost parameter.

Converting the zero-mode solution (5.20)-(5.23) into the Chern-Simons formulation and using formula (4.34) then yields the entropy

$$
S=\frac{2 \pi L|\hat{\eta}|}{4 G_{N}}
$$

where independence of the AdS radius follows from the fact that the connection given by (5.4a) has no non-zero component along any of the $M_{n}$ 's. This agrees with the microscopic result (5.17).

The macroscopic free energy compatible with the first law $\mathrm{d} F=-S \mathrm{~d} T-P_{0} \mathrm{~d} \hat{\eta}$ is given by

$$
F(T, \hat{\eta})=H\left(S, P_{0}\right)-T S-P_{0} \hat{\eta}=-2 \pi L k|\hat{\eta}| T+k L \hat{\eta}^{2} / \ell
$$

where $H$ is given by the zero mode charge $T=a \eta$ through (4.2), $H=k L a \eta=-F$, and $P_{0}$ is given by the zero mode charge $P=a$ through the right eq. (3.13), $P_{0}=k L a$. The result (5.25) also coincides with the microscopic one (5.16).

\section{Discussion}

In this final section we highlight some of the unusual features that we unraveled in our quest for near horizon holography. We add some comments, explanations and possible resolutions of the open issues.

\subsection{Role of retarded time}

Let us summarize and discuss aspects of the dependence of Rindler acceleration on retarded time. (Note that our whole paper can easily be sign-flipped to advanced time $v$, which may be useful in some applications.) 
Rindler acceleration depends on retarded time. We started with the ansatz (1.2) since we wanted a state-dependent Rindler acceleration to accommodate a state-dependent temperature. We left it open whether Rindler acceleration $a$ was a function of retarded time $u$, spatial coordinate $x$ or both. The Einstein equations forced us to conclude that Rindler acceleration can depend on retarded time only. We give now a physical reason why this should be expected. Namely, if the zeroth law of black hole mechanics holds then surface gravity (and thus Rindler acceleration) must be constant along the horizon. In particular, it cannot depend on $x$. If the horizon changes, e.g. due to emission of Hawking quanta or absorption of matter, then Rindler acceleration can change, which makes the dependence on $u$ natural, much like the corresponding dependence of Bondi mass on the lightlike time.

Retarded time is periodic. While many of our results are actually independent of the choice (2.23), it was still a useful assumption for several purposes, e.g. the introduction of Fourier modes. For some physical observables it is possible to remove this assumption by taking the limit $L \rightarrow \infty$. We shall provide an important example in section 6.2 below.

Boundary currents are integrated over retarded time. If we wanted our theory to be non-empty we could not use the standard definition of canonical charges integrated over space, but instead had to consider boundary currents integrated over retarded time. We have no further comments on this issue, except for pointing out that in four dimensions, a bilinear in the Bondi news ${ }^{9}$ is integrated over retarded time in order to yield the ADM mass. Thus, despite of the clash with the usual Hamilton formulation we believe that we have made here the most natural choice, given our starting point.

\subsection{Rindler entropy?}

Let us finally take a step back and try to connect with our original aim of setting up Rindler holography and microscopically calculating the Rindler entropy [52]. We summarize in appendix B results for Rindler thermodynamics and Rindler entropy, which match the near horizon results of BTZ black holes and flat space cosmologies.

We consider a limiting procedure starting with our result for entropy (4.33). We are interested in a limit where simultaneously the compactification length $L$ in (2.23) tends to infinity, the boost parameter $\eta$ tends to zero, the length of the spatial cycle $x$ appears in the entropy and all unusual factors of $i$ are multiplied by something infinitesimal. In other words, we try to construct a limit towards Rindler entropy (B.12).

Consider the identifications (4.3) with a complexified $\beta \rightarrow \beta_{0}+2 \pi i L$ and split them into real and imaginary parts:

$$
\operatorname{Re}:(u, x) \sim(u, x+2 \pi L \eta) \sim(u+2 \pi L, x) \quad \operatorname{Im}:(u, x) \sim\left(u+i \beta_{0}, x-i \beta_{0} \eta\right)
$$

The rationale behind this shift is that the real part of the periodicities untwists. As in appendix B we call the (real) length of the $x$-cycle $\tilde{L}$ and thus have the relation

$$
\tilde{L}=2 \pi L \eta \text {. }
$$

\footnotetext{
${ }^{9}$ Bondi news is the outgoing flux of gravitational radiation, see e.g. [105] and references therein.
} 
Therefore, taking the decompactification limit for retarded time, $L \rightarrow \infty$, while keeping fixed $\tilde{L}$ simultaneously achieves the desired $\eta \rightarrow 0$, so that the periodicities (6.1) in this limit simplify to

$$
\operatorname{Re}:(u, x) \sim(u, x+\tilde{L}) \quad \operatorname{Im}:(u, x) \sim\left(u+i \beta_{0}, x\right)
$$

which, if interpreted as independent periodicities, are standard relations for non-rotating horizons at inverse temperature $\beta_{0}$ and with a length of the spatial cycle given by $\tilde{L}$. Apart from taking limits our only manipulation was to shift the inverse temperature $\beta$ in the complex plane. Thus, any observable that is independent from temperature should remain unaffected by such a shift; moreover, the "compactification" of $\beta$ along the imaginary axis is then undone by taking the decompactification limit $L \rightarrow \infty$. We conclude from this that entropy $S$ from (4.33) should have a smooth limit under all the manipulations above and hopefully yield the Rindler result (B.12). This is indeed the case:

$$
\lim _{L \rightarrow \infty, \eta \rightarrow \tilde{L} /(2 \pi L)}\left(\lim _{\beta \rightarrow \beta_{0}+2 \pi i L} S\right)=\frac{\tilde{L}}{4 G_{N}} .
$$

Thus, we recover the usual Bekenstein-Hawking entropy law as expected from Rindler holography. In this work we have also provided a Cardy-like derivation of this result. A different singular limit was considered in [106], where the Rindler entropy (6.4) was derived from a Cardy formula for holographic hyperscaling theories.

\subsection{Other approaches}

Relation to BMS/ultrarelativistic CFT. Using our quadratic map (3.24) of warped CFT generators to $\mathrm{BMS}_{3}$ generators together with the result (3.23) for the central charge, we now check what microstate counting would be given by an ultrarelativistic CFT (or equivalently a Galilean CFT) [20,21]. Using the "angular momentum" $h_{L}=a \eta$, the "mass" $h_{M}=a^{2} /(2 k)$, the central charge $c_{M}=12 k$ with $k=1 /\left(4 G_{N}\right)$, and introducing an extra factor of $L$ to accommodate our periodicity (2.23), the ultrarelativistic Cardy formula gives

$$
S_{\mathrm{UCFT}}=2 \pi L\left|h_{L}\right| \sqrt{\frac{c_{M}}{24 h_{M}}}=2 \pi L|a \eta| \sqrt{\frac{k^{2}}{a^{2}}}=\frac{2 \pi L|\eta|}{4 G_{N}} .
$$

This entropy thus coincides with the warped CFT entropy (4.12), and matches the gravity result (4.33).

Other Rindler-type boundary conditions. While finishing this work the paper [107] appeared which proposes alternative Rindler boundary conditions, motivated partly by Hawking's claim that the information loss paradox can be resolved by considering the supertranslation of the horizon caused by ingoing particles [108, 109]. ${ }^{10}$ (See also [111, 112].) In [107] the state-dependent functions depend on the spatial coordinate and thus allow for standard canonical charges. The corresponding Rindler acceleration (and thus

\footnotetext{
${ }^{10}$ After posting this paper, a more detailed account of the relationship between near horizon properties and supertranslations was posted by Hawking, Perry and Strominger [110], where they argue that supertranslations generate "soft hair" on black holes, where "soft" means "zero energy".
} 
temperature) is state-independent and the asymptotic symmetry algebra has no central extension. We checked that the Rindler acceleration of that paper can be made statedependent, but in accordance with our discussion in section 6.1 it cannot depend on the spatial coordinate; only dependence on retarded/advanced time is possible. Thus, we believe that if one wants to allow for a state-dependent temperature in Rindler holography the path described in the present work is unavoidable.

Generalizations. We finish by mentioning a couple of interesting generalizations that should allow in several cases straightforward applications of our results, like generalizations to higher derivative theories of (massive or partially massless) gravity, theories that include matter and theories in higher dimensions. In particular, it would be interesting to generalize our discussion to topologically massive gravity $[113,114]$ in order to see how the entropy computation would be affected.

\section{Acknowledgments}

We are grateful to Gaston Giribet for collaboration and for sharing his insights on Rindler holography during the first two years of this project (August 2013 - June 2015). In addition, we thank Glenn Barnich, Diego Hofman and Friedrich Schöller for discussions.

HA was supported in part by the Dutch stichting voor Fundamenteel Onderzoek der Materie (FOM) and in part by the Iranian National Science Foundation (INSF). SD is a Research Associate of the Fonds de la Recherche Scientifique F.R.S.-FNRS (Belgium). He is supported in part by the ARC grant "Holography, Gauge Theories and Quantum Gravity Building models of quantum black holes", by IISN - Belgium (convention 4.4504.15) and benefited from the support of the Solvay Family. He thanks Gaston Giribet and Buenos Aires University for hosting him while this work was in progress. DG was supported by the Austrian Science Fund (FWF), projects Y 435-N16, I 952-N16, I 1030-N27 and P 27182N27, and by the program Science without Borders, project CNPq-401180/2014-0. DG also thanks Buenos Aires University for hosting a research visit, supported by OeAD project AR 09/2013 and CONICET, where this work was commenced. BO was supported by the Fund for Scientific Research-FNRS Belgium (under grant number FC-95570) and by a research fellowship of the Wiener-Anspach Foundation.

\section{A On representations of the warped Virasoro group}

This appendix is devoted to certain mathematical considerations regarding the warped Virasoro group. They are motivated by the questions encountered in section 3 of this paper, although they are also interesting in their own right. First, in subsection A.1 we study the coadjoint representation of the warped Virasoro group, which is needed to derive formulas (3.17)-(3.18) for the transformation law of the stress tensor. Then, in subsection A.2 we classify all irreducible, unitary representations of this group with vanishing $u(1)$ level using the method of induced representations. We assume throughout that the coordinate $u$ is $2 \pi L$-periodic. 


\section{A.1 Coadjoint representation}

We call warped Virasoro group, denoted $\hat{G}$, the general central extension of the group

$$
G=\operatorname{Diff}^{+}\left(S^{1}\right) \ltimes \mathrm{C}^{\infty}\left(S^{1}\right),
$$

where the notation is the same as in (3.3) up to the replacement of $\mathbb{R}$ by $S^{1}$ [so that in particular $f$ satisfies property (3.15)]. In this subsection we display an explicit definition of $\hat{G}$ and work out its adjoint and coadjoint representations, using a $2 \pi L$-periodic coordinate $u$ to parametrize the circle. We refer to $[104,115]$ for more details on the Virasoro group and its cohomology.

Since the differentiable, real-valued second cohomology space of $G$ is threedimensional $[104,116]$, there are exactly three central extensions to be taken into account when defining $\hat{G}$; in other words, $\hat{G} \cong G \times \mathbb{R}^{3}$ as manifolds. Accordingly, elements of $\hat{G}$ are pairs $(f, p)$ belonging to $G$, supplemented by triples of real numbers $(\lambda, \mu, \nu)$. The group operation in $\hat{G}$ is

$$
\begin{aligned}
& \left(f_{1}, p_{1} ; \lambda_{1}, \mu_{1}, \nu_{1}\right) \cdot\left(f_{2}, p_{2} ; \lambda_{2}, \mu_{2}, \nu_{2}\right)= \\
= & \left(f_{1} \circ f_{2}, p_{1}+\sigma_{f_{1}} p_{2} ; \lambda_{1}+\lambda_{2}+B\left(f_{1}, f_{2}\right), \mu_{1}+\mu_{2}+C\left(f_{1}, p_{2}\right), \nu_{1}+\nu_{2}+D\left(f_{1}, p_{1}, p_{2}\right)\right),
\end{aligned}
$$

where $\sigma$ is the action (3.4) while $B, C$ and $D$ are non-trivial 2-cocycles on $G$ given explicitly by

$$
\begin{aligned}
B\left(f_{1}, f_{2}\right) & =-\frac{1}{48 \pi} \int_{S^{1}} \ln \left(f_{1}^{\prime} \circ f_{2}\right) \mathrm{d} \ln \left(f_{2}^{\prime}\right), \\
C\left(f_{1}, p_{2}\right) & =-\frac{1}{2 \pi} \int_{S^{1}} p_{2} \cdot \mathrm{d} \ln \left(f_{1}^{\prime}\right), \\
D\left(f_{1}, p_{1}, p_{2}\right) & =-\frac{1}{4 \pi} \int_{S^{1}} p_{1} \cdot \mathrm{d}\left(\sigma_{f_{1}} p_{2}\right) .
\end{aligned}
$$

In particular, $B$ is the standard Bott-Thurston cocycle [115] defining the Virasoro group.

Adjoint representation and Lie brackets. To write down an explicit formula for the coadjoint representation of the warped Virasoro group, we first need to work out the adjoint representation, which acts on the Lie algebra $\hat{\mathfrak{g}}$ of $\hat{G}$. As follows from the definition of $\hat{G}$, that algebra consists of 5 -tuples $(t, p ; \lambda, \mu, \nu)$ where $t=t(u) \frac{\partial}{\partial u}$ is a vector field on the circle, $p=p(u)$ is a function on the circle, and $\lambda, \mu, \nu$ are real numbers. The adjoint representation of $\hat{G}$, which we will denote as $\mathrm{Ad}$, is then defined as

$$
\begin{aligned}
& \operatorname{Ad}_{\left(f, p_{1} ; \lambda_{1}, \mu_{1}, \nu_{1}\right)}\left(t, p_{2} ; \lambda_{2}, \mu_{2}, \nu_{2}\right)= \\
= & \left.\frac{\mathrm{d}}{\mathrm{d} \epsilon}\left[\left(f, p_{1} ; \lambda_{1}, \mu_{1}, \nu_{1}\right) \cdot\left(e^{\epsilon t}, \epsilon p_{2} ; \epsilon \lambda_{2}, \epsilon \mu_{2}, \epsilon \nu_{2}\right) \cdot\left(f, p_{1} ; \lambda_{1}, \mu_{1}, \nu_{1}\right)^{-1}\right]\right|_{\epsilon=0}
\end{aligned}
$$

where $e^{\epsilon t}$ is to be understood as an infinitesimal diffeomorphism $e^{\epsilon t}(u)=u+\epsilon t(u)+\mathcal{O}\left(\epsilon^{2}\right)$. Given the group operation (A.2), it is easy to verify that the central terms $\lambda_{1}, \mu_{1}$ and $\nu_{1}$ play 
a passive role, so we may simply set them to zero and write $\operatorname{Ad}_{(f, p ; \lambda, \mu, \nu)} \equiv \operatorname{Ad}_{(f, p)}$. Using multiple Taylor expansions and integrations by parts, the right-hand side of (A.6) yields

$$
\begin{aligned}
\operatorname{Ad}_{\left(f, p_{1}\right)}\left(t, p_{2} ; \lambda, \mu, \nu\right)= & \left(\operatorname{Ad}_{f} t, \sigma_{f} p_{2}+\Sigma_{\mathrm{Ad}_{f} t} p_{1} ; \lambda-\frac{1}{24 \pi} \int_{0}^{2 \pi L} \mathrm{~d} u t(u)\{f ; u\},\right. \\
& \mu-\frac{1}{2 \pi} \int p_{2} \cdot \mathrm{d} \ln \left(f^{\prime}\right)+\left.\frac{1}{2 \pi} \int_{0}^{2 \pi L} \mathrm{~d} u t(u)\left[\left(p_{1} \circ f\right)^{\prime \prime}-\left(p_{1} \circ f\right)^{\prime} \frac{f^{\prime \prime}}{f^{\prime}}\right]\right|_{u}, \\
& \left.\nu-\frac{1}{2 \pi} \int p_{1} \cdot \mathrm{d}\left(\sigma_{f} p_{2}\right)+\frac{1}{4 \pi} \int_{0}^{2 \pi L} \mathrm{~d} u t(u)\left[\left(p_{1} \circ f\right)^{\prime}(u)\right]^{2}\right),
\end{aligned}
$$

where prime denotes differentiation with respect to $u$. Let us explain the meaning of the symbols appearing here:

- The symbol Ad on the right-hand side denotes the adjoint representation of the group $\operatorname{Diff}^{+}\left(S^{1}\right)$ :

$$
\left.\left(\operatorname{Ad}_{f} t\right)(u) \equiv \frac{\mathrm{d}}{\mathrm{d} \epsilon}\left[f \circ e^{\epsilon t} \circ f^{-1}(u)\right]\right|_{\epsilon=0}=f^{\prime}\left(f^{-1}(u)\right) \cdot t\left(f^{-1}(u)\right) .
$$

The far right-hand side of this equation should be seen as the component of a vector field $\left(\operatorname{Ad}_{f} t\right)(u) \frac{\mathrm{d}}{\mathrm{d} u}$. Equivalently,

$$
\left(\operatorname{Ad}_{f} t\right)(f(u))=f^{\prime}(u) \cdot t(u)
$$

which is the usual transformation law of vector fields on the circle under diffeomorphisms.

- The quantity $\{f ; u\}$ is the Schwarzian derivative of the diffeomorphism $f$ evaluated at $u$ :

$$
\left.\{f ; u\} \equiv\left[\frac{f^{\prime \prime \prime}}{f^{\prime}}-\frac{3}{2}\left(\frac{f^{\prime \prime}}{f^{\prime}}\right)^{2}\right]\right|_{u} .
$$

- The symbol $\Sigma$ denotes the differential of the action $\sigma$ of $\operatorname{Diff}^{+}\left(S^{1}\right)$ on $C^{\infty}\left(S^{1}\right)$. Explicitly, if $t$ is a vector field on the circle and if $p \in C^{\infty}\left(S^{1}\right)$,

$$
\left(\Sigma_{t} p\right)(u) \equiv-\left.\frac{\mathrm{d}}{\mathrm{d} \epsilon}\left[\left(\sigma_{e^{\epsilon t}} p\right)(u)\right]\right|_{\epsilon=0}=t(u) \cdot p^{\prime}(u) .
$$

It is easily verified, upon considering an infinitesimal diffeomorphism $f$ and an infinitesimal function $p_{1}$, that the Lie brackets defined by this adjoint representation coincide with the standard brackets of a centrally extended warped Virasoro algebra. More precisely, upon defining the generators

$$
T_{n} \equiv\left(L e^{i n u / L} \frac{\partial}{\partial u}, 0 ; 0,0,0\right) \quad P_{n} \equiv\left(0, e^{i n u / L} ; 0,0,0\right)
$$


and the central charges

$$
Z_{1} \equiv(0,0 ; 1,0,0) \quad Z_{2} \equiv(0,0 ; 0,1,0) \quad Z_{3} \equiv(0,0 ; 0,0,1)
$$

the Lie brackets defined by

$$
\left[\left(t_{1}, p_{1} ; \lambda_{1}, \mu_{1}, \nu_{1}\right),\left(t_{2}, p_{2} ; \lambda_{2}, \mu_{2}, \nu_{2}\right)\right] \equiv-\left.\frac{\mathrm{d}}{\mathrm{d} \epsilon}\left[\operatorname{Ad}_{\left(e^{\left.\epsilon t_{1}, \epsilon p_{1} ; \epsilon \lambda_{1}, \epsilon \mu_{1}, \epsilon \nu_{1}\right)}\right.}\left(t_{2}, p_{2} ; \lambda_{2}, \mu_{2}, \nu_{2}\right)\right]\right|_{\epsilon=0}
$$

turn out to read

$$
\begin{aligned}
i\left[T_{n}, T_{m}\right] & =(n-m) T_{n+m}+\frac{Z_{1}}{12} n^{3} \delta_{n+m, 0} \\
i\left[T_{n}, P_{m}\right] & =-m P_{n+m}-i Z_{2} n^{2} \delta_{n+m, 0} \\
i\left[P_{n}, P_{m}\right] & =Z_{3} n \delta_{n+m, 0} .
\end{aligned}
$$

Here we recognize the centrally extended algebra (3.16), up to the fact that the central charges $Z_{i}$ are written as operators; eventually they will be multiples of the identity, with coefficients $c, \kappa$ and $K$ corresponding to $Z_{1}, Z_{2}$ and $Z_{3}$ respectively.

Coadjoint representation. The coadjoint representation of $\hat{G}$ is the dual of the adjoint representation, and coincides with the finite transformation laws of the functions $T$ and $P$ introduced in (3.10) [i.e. the stress tensor and the $u(1)$ current]. Explicitly, the dual $\hat{\mathfrak{g}}^{*}$ of the Lie algebra $\hat{\mathfrak{g}}$ consists of 5 -tuples

$$
(T, P ; c, \kappa, K)
$$

where $T=T(u) \mathrm{d} u \otimes \mathrm{d} u$ is a quadratic density on the circle, $P=P(u) \mathrm{d} u$ is a one-form on the circle, and $c, \kappa, K$ are real numbers - those are the values of the various central charges. We define the pairing of $\hat{\mathfrak{g}}^{*}$ with $\hat{\mathfrak{g}}$ by ${ }^{11}$

$$
\langle(T, P ; c, \kappa, K),(t, p ; \lambda, \mu, \nu)\rangle \equiv \frac{k}{2 \pi} \int_{0}^{2 \pi L} \mathrm{~d} u(T(u) t(u)+P(u) p(u))+c \lambda+\kappa \mu+K \nu,
$$

so that it coincides, up to central terms, with the definition of surface charges (3.11). Note that here $c$ is the usual Virasoro central charge, $K$ is the $u(1)$ level and $\kappa$ is the twist central charge appearing in (3.16). The coadjoint representation $\mathrm{Ad}^{*}$ of $\hat{G}$ is defined by

$$
\operatorname{Ad}_{(f, p)}^{*}(T, P ; c, \kappa, K) \equiv(T, P ; c, \kappa, K) \circ \operatorname{Ad}_{(f, p)^{-1}} .
$$

Using the explicit form (A.7) of the adjoint representation, one can read off the transformation law of each component in (A.18). The result is

$$
\operatorname{Ad}_{(f, p)}^{*}(T, P ; c, \kappa, K)=\left(\operatorname{Ad}_{(f, p)}^{*} T, \operatorname{Ad}_{(f, p)}^{*} P ; c, \kappa, K\right)
$$

\footnotetext{
${ }^{11}$ As usual $[115,117]$, what we call the "dual space" here is really the smooth dual space, i.e. the space of regular distributions on the space of functions or vector fields on the circle.
} 
(i.e. the central charges are left invariant by the action of $G$ ), where $\operatorname{Ad}_{(f, p)}^{*} T$ and $\operatorname{Ad}_{(f, p)}^{*} P$ are a quadratic density and a one-form on the circle (respectively) whose components, evaluated at $f(u)$, are

$$
\begin{aligned}
& \left.\quad \operatorname{Ad}_{(f, p)}^{*} T\right)(f(u))=\frac{1}{\left(f^{\prime}(u)\right)^{2}} \times \\
& \quad \times\left[T(u)+\frac{c}{12 k}\{f ; u\}-P(u)(p \circ f)^{\prime}(u)-\frac{\kappa}{k}(p \circ f)^{\prime \prime}(u)+\frac{K}{2 k}\left((p \circ f)^{\prime}(u)\right)^{2}\right]
\end{aligned}
$$

and

$$
\left(\operatorname{Ad}_{(f, p)}^{*} P\right)(f(u))=\frac{1}{f^{\prime}(u)}\left[P(u)+\frac{\kappa}{k} \frac{f^{\prime \prime}(u)}{f^{\prime}(u)}-\frac{K}{k}(p \circ f)^{\prime}(u)\right] .
$$

These are the transformation laws displayed in (3.17)-(3.18), with $\operatorname{Ad}_{(f, p)}^{*} T \equiv \tilde{T}$ and $\operatorname{Ad}_{(f, p)}^{*} P \equiv \tilde{P}$. They reduce to the transformations of a standard warped CFT [86] for $\kappa=0$. In the Rindler case, however, $c=K=0$ and $\kappa=k$ is non-zero.

The transformation law of the function $\eta(u)$ in (3.6) under finite asymptotic symmetry transformations can be worked out in a much simpler way. Indeed, it is easily verifed that the left formula in (3.6) reproduces (2.30) for infinitesimal transformations, and is compatible with the group operation (3.5) of the warped Virasoro group. One can also check that the transformation laws (3.19) follow from (3.6) and the definition (3.10).

\section{A.2 Induced representations}

As indicated by the imaginary vacuum values (4.9) (which are actually fairly common in the world of warped CFT's [86]), the asymptotic symmetry group is not represented in a unitary way in quasi-Rindler holography. Nevertheless, since the standard interpretation of symmetries in quantum mechanics requires unitarity [118], it is illuminating to study unitary representations of the warped Virasoro group. Here we classify such representations for the case of vanishing Kac-Moody level $K$, but non-vanishing twist $\kappa$. As in the case of the Euclidean, Poincaré or BMS groups, the semi-direct product structure (A.1) [or similarly (3.9)] is crucial; indeed, all irreducible unitary representations of such a group are induced à la Wigner [119-122]. We refer to [123, 124] for more details on induced representations and we mostly use the notations of [26-28].

A lightning review of induced representations. The construction of induced representations of the warped Virasoro group $\hat{G}$ with vanishing $u(1)$ level follows the same steps as for the Poincaré group [122, 125] or the $\mathrm{BMS}_{3}$ group [26, 27]. One begins by identifying the dual space of the Abelian group $\mathrm{C}^{\infty}\left(S^{1}\right)$, which in the present case consists of currents $P(u) \mathrm{d} u$. [The elements of this dual space are typically called "momenta", and our notation $P(u)$ is consistent with that terminology.] One then defines the orbit $\mathcal{O}_{P}$ and the little group $G_{P}$ of $P=P(u) \mathrm{d} u$ as

$$
\mathcal{O}_{P} \equiv\left\{f \cdot P \mid f \in \operatorname{Diff}^{+}\left(S^{1}\right)\right\} \quad \text { and } \quad G_{P} \equiv\left\{f \in \operatorname{Diff}^{+}\left(S^{1}\right) \mid f \cdot P=P\right\},
$$

where the action of $f$ on $P$ is given by (3.20). Then, given an orbit $\mathcal{O}_{P}$ and an irreducible, unitary representation $\mathcal{R}$ of its little group $G_{P}$ in some Hilbert space $\mathcal{E}$, the corresponding 
induced representation $\mathcal{T}$ acts on square-integrable wave functions $\Psi: \mathcal{O}_{P} \rightarrow \mathcal{E}: q \mapsto \Psi(q)$ according to $[123,124]$

$$
(\mathcal{T}[(f, p)] \Psi)(q) \equiv\left[\rho_{f^{-1}}(q)\right]^{1 / 2} e^{i\langle q, p\rangle} \mathcal{R}\left[g_{q}^{-1} f g_{f^{-1} \cdot q}\right] \Psi\left(f^{-1} \cdot q\right),
$$

where $(f, p)$ belongs to $\operatorname{Diff}^{+}\left(S^{1}\right) \ltimes C^{\infty}\left(S^{1}\right)$. Let us briefly explain the terms of this equation:

- The real, positive function $\rho_{f}$ on $\mathcal{O}_{P}$ denotes the Radon-Nikodym derivative of the measure used to define the scalar product of wavefunctions. It is an "anomaly" that takes the value $\rho_{f}=1$ for all $f$ when the measure is invariant, but otherwise depends on $f$ and on the point $q$ at which it is evaluated. In simple cases (e.g. the Poincaré group), the measure is invariant and $\rho_{f}(q)=1$ for all $f$ and all $q \in \mathcal{O}_{P}$.

- The operator $\mathcal{R}\left[g_{q}^{-1} f g_{f^{-1 . q}}\right]$ is a "Wigner rotation": it is the transformation corresponding to $f$ in the space of spin degrees of freedom of the representation $\mathcal{T}$. We denote by $g_{q}$ the "standard boost" associated with $q$, that is, a group element such that $g_{q} \cdot P=q$. For scalar representations, $\mathcal{R}$ is trivial and one may simply forget about the Wigner rotation.

The classification of irreducible, unitary representations of the central extension of $\operatorname{Diff}^{+}\left(S^{1}\right) \ltimes C^{\infty}\left(S^{1}\right)$ with vanishing Kac-Moody level then amounts to the classification of all possible orbits (A.23) and of all unitary representations of the corresponding little groups.

Induced representations of $\hat{\boldsymbol{G}}$. Our goal now is to classify all irreducible, unitary representations of the warped Virasoro group with vanishing Kac-Moody level, under the assumption that there exists a quasi-invariant measure on all the orbits (see [126-129] for the construction of such measures). According to the lightning review just displayed, this amounts to the classification of orbits, as defined in (A.23). We start with two preliminary observations:

1. For any constant current $P(u)=P_{0}=$ const, the little group $G_{P}$ consists of rigid time translations $f(u)=u+u_{0}$.

2. The charge $Q[P]$ defined as

$$
Q[P] \equiv \frac{k}{2 \pi} \int_{0}^{2 \pi L} \mathrm{~d} u P(u)
$$

is constant along any coadjoint orbit of the warped Virasoro group, regardless of the values of the central charges $c, \kappa$ and $K$. In other words, for any current $P$, any (orientation-preserving) diffeomorphism $f$ of the circle and any function $p$, the zero-mode of $P$ is left invariant by the coadjoint action:

$$
Q\left[\operatorname{Ad}_{(f, p)}^{*} P\right]=Q[P]
$$


where $\operatorname{Ad}_{(f, p)}^{*} P$ is given by (3.18). This result holds, in particular, in the case $c=$ $K=0$ that we wish to study, and corresponds physically to the fact that the average value of acceleration is invariant under asymptotic symmetries.

The proof of both results is straightforward, as they can be verified by brute force. In fact, they follow from a stronger statement: it turns out that the orbits $\mathcal{O}_{P}$ foliate the space of currents into hyperplanes of constant $Q[P]$, so that any current $P(u)$ can be brought to a constant by acting with a diffeomorphism. To prove this, note that constancy of $Q[P]$ implies that that constant, if it exists, coincides with the zero-mode $P_{0}$ of $P(u)$. The question thus boils down to whether or not there exists an orientation-preserving diffeomorphism $f$ such that

$$
\left.(f \cdot P)\right|_{f(u)} \stackrel{(3.6)}{=} \frac{1}{f^{\prime}(u)}\left[P(u)+\frac{\kappa}{k} \frac{f^{\prime \prime}(u)}{f^{\prime}(u)}\right] \stackrel{!}{=} P_{0} .
$$

This condition is equivalent to an inhomogeneous first-order differential equation for $1 / f^{\prime}$, whose solution is

$$
\frac{1}{f^{\prime}(u)}=A \exp \left[\frac{k}{\kappa} \int_{0}^{u} \mathrm{~d} v P(v)\right]-\frac{k}{\kappa} P_{0} \int_{0}^{u} \mathrm{~d} t \exp \left[\frac{k}{\kappa} \int_{t}^{u} \mathrm{~d} v P(v)\right]
$$

where $A$ is a real parameter. Since $u$ is assumed to be $2 \pi L$-periodic, this function must be $2 \pi L$-periodic as well. This selects a unique solution (and such a solution exists for any value of $P_{0}$ ), meaning that, for any $P(u)$, there always exists a diffeomorphism $f$ of the circle such that $f \cdot P$ be a constant, ${ }^{12}$ furthermore, that diffeomorphism is uniquely specified by $P(u)$ up to a rigid time translation.

This proves that all orbits $\mathcal{O}_{P}$ are hyperplanes specified by the value of the charge (A.25), in accordance with the fact that $P_{0}$ is a Casimir operator in (3.16). One can then apply the usual machinery of induced representations to the warped Virasoro group, and compute, for instance, the associated characters along the lines of [28]; however, as the interpretation of these characters in the present context is unclear, we refrain from displaying them.

Physical properties of unitary representations. We have just seen that unitary representations of the warped Virasoro group [with vanishing $u(1)$ level] can be classified according to the possible values of the Casimir operator $P_{0}$ in (3.16). Accordingly, the orbits (A.23) are affine hyperplanes with constant zero-modes embedded in the space of currents $P(u)$. In particular, each orbit contains exactly one constant representative. The physical meaning of this statement is that any (generally time-dependent) acceleration $a(u)$ can be brought to a constant, $a_{0}$, by using a suitable reparametrization of time that

\footnotetext{
${ }^{12}$ An alternative way to prove the same result is to recall the modified Sugawara construction (3.21), by which a coadjoint orbit of the Virasoro group with non-negative energy is associated with each orbit $\mathcal{O}_{P}$. Since the only Virasoro orbits with non-negative energy are orbits of constants, we know that there always exists a diffeomorphism $f$ that brings a given Sugawara stress tensor (3.21) into a constant, which in turn brings the corresponding current $P$ to a constant.
} 
preserves $2 \pi L$-periodicity. Furthermore, $a_{0}$ coincides with the Fourier zero-mode of $a(u)$. Note that, with the requirement of $2 \pi L$-periodicity, it is no longer true that any timedependent acceleration $a(u)$ can be mapped on $\tilde{a}=0$ because the diffeomorphisms defined by (3.7) generally do not preserve that requirement.

Having classified the orbits, we know, in principle, the irreducible unitary representations of the warped Virasoro group at vanishing Kac-Moody level. In three spacetime dimensions, these representations describe the Hilbert space of metric fluctuations around the background specified by the orbit $\mathcal{O}_{P}$ and the representation $\mathcal{R}[26,27,29,101,130]$, as follows from the fact that the phase space coincides with the coadjoint representation of the asymptotic symmetry group. For instance, an induced representation of $\hat{G}$ specified by an orbit $\mathcal{O}_{P}$ and the trivial representation of the little group gives the Hilbert space of metric fluctuations around the background

$$
\mathrm{d} s^{2}=-2 P(u) r \mathrm{~d} u^{2}-2 \mathrm{~d} u \mathrm{~d} r+\mathrm{d} x^{2} .
$$

Here $u$ is still understood as a $2 \pi L$-periodic coordinate; in particular, the solution at $a=0$ is not Minkowski spacetime because of that identification.

Note that, in any unitary representation of the type just described, the eigenvalues of the Hamiltonian $T_{0}$ are unbounded from below (and from above). There is thus a trade-off between unitarity and boundedness of energy: if we insist that the representation be unitary, then it is a (direct integral of) induced representation(s), and energy is unbounded both from below and from above; conversely, if we insist that energy be bounded from below, then the asymptotic symmetry group cannot act unitarily on the Hilbert space of the putative dual theory. This property has actually been observed in representations of the Galilean Conformal Algebra in two dimensions, $\mathfrak{g c a}_{2}$, and its higher-spin extensions $[24,25,131]$. Indeed, when $T_{0}$ is interpreted as the Hamiltonian, demanding that energy be bounded from below amounts to considering highest-weight representations of the symmetry algebras (3.14) or (3.25), the highest weight being the lowest eigenvalue of $T_{0}$ in the space of the representation. This representation is easily seen to be non-unitary when the central charge of the mixed commutator is non-zero. We stress, however, that in the more common interpretation of the warped Virasoro group [86] where $P_{0}$ plays the role of the Hamiltonian, there is no such conflict between unitarity and boundedness of energy.

\section{B Rindler thermodynamics}

We have not found a holographic setup that leads to Rindler thermodynamics, but it is still of interest in its own right to consider it. In this appendix we describe Rindler thermodynamics in a non-holographic context and show that it recovers the near horizon thermodynamics of BTZ black holes and flat space cosmologies, in the sense that temperatures and entropies agree with each other. If a consistent version of Rindler holography exists, it should reproduce the results of this appendix (see also [52]).

The main change as compared to the main text is that the periodicities in the Euclidean coordinates are no longer given by (4.27), but instead by

$$
\left(t_{\mathrm{E}}, y\right) \sim\left(t_{\mathrm{E}}+\beta, y-i \beta \eta\right) \sim\left(t_{\mathrm{E}}, y+\tilde{L}\right)
$$


where $\tilde{L}$ is now the periodicity of the spatial coordinate $y$ and $\beta, \eta$ coincide with the quasi-Rindler parameters. So in Rindler thermodynamics we do not identify retarded time periodically, which is the key difference to quasi-Rindler thermodynamics.

\section{B.1 Rindler horizon and temperature}

The Euclidean metric (4.26) has a center at $\rho=0$, corresponding to the Rindler horizon in Lorentzian signature. In order for this center to be smooth, the Euclidean time $t_{\mathrm{E}} \sim$ $t_{\mathrm{E}}+\beta$ has to have a periodicity $\beta=2 \pi / a$. Interpreting the inverse of this periodicity as temperature yields the expected Unruh temperature $[50,51]$

$$
T=\frac{a}{2 \pi} \text {. }
$$

The same result is obtained from surface gravity. Note that the Unruh temperature (B.2) is independent of the boost parameter $\eta$.

There is yet another way to determine the Unruh temperature, namely starting from rotating (non-extremal) BTZ and taking the near horizon limit. The rotating BTZ metric $[4,5]$

$$
\mathrm{d} s^{2}=-\frac{\left(r^{2}-r_{+}^{2}\right)\left(r^{2}-r_{-}^{2}\right)}{\ell^{2} r^{2}} \mathrm{~d} t^{2}+\frac{\ell^{2} r^{2}}{\left(r^{2}-r_{+}^{2}\right)\left(r^{2}-r_{-}^{2}\right)} \mathrm{d} r^{2}+r^{2}\left(\mathrm{~d} \varphi-\frac{r_{+} r_{-}}{\ell r^{2}} \mathrm{~d} t\right)^{2}
$$

leads to a Hawking temperature

$$
T_{H}=\frac{r_{+}^{2}-r_{-}^{2}}{2 \pi r_{+} \ell^{2}} .
$$

Now take the near horizon limit by defining $\rho=\left(r^{2}-r_{+}^{2}\right) /\left(2 r_{+}\right)$and dropping higher order terms in $\rho$, which gives

$$
\mathrm{d} s^{2}=-2 a \rho \mathrm{d} t^{2}+\frac{\mathrm{d} \rho^{2}}{2 a \rho}+(\mathrm{d} x+\eta \mathrm{d} t)^{2}
$$

with

$$
a=\frac{\hat{r}_{+}^{2}-\hat{r}_{-}^{2} / \ell^{2}}{\hat{r}_{+}}, \quad \eta=-\hat{r}_{-}, \quad x=\hat{r}_{+} \ell^{2} \varphi,
$$

where $\hat{r}_{+}=r_{+} / \ell^{2}$ and $\hat{r}_{-}=r_{-} / \ell$. Note that in the limit of infinite AdS radius, $\ell \rightarrow \infty$, we keep fixed the rescaled parameters $\hat{r}_{ \pm}$, and the coordinate $x$ decompactifies. [We then recompactify by imposing (B.1).] The Hawking temperature $T_{H}$ can be rewritten as

$$
T_{H}=\frac{\hat{r}_{+}^{2}-\hat{r}_{-}^{2} / \ell^{2}}{2 \pi \hat{r}_{+}}=\frac{a}{2 \pi}
$$

and thus coincides with the Unruh temperature (B.2). Besides verifying this expected result, the calculation above provides expressions for the Rindler parameters $a$ and $\eta$ in terms of BTZ parameters $r_{ \pm}$, which can be useful for other consistency checks as well.

Essentially the same conclusion holds for flat space cosmologies $[6,7]$, whose metric reads

$$
\mathrm{d} s^{2}=r_{+}^{2}\left(1-\frac{r_{0}^{2}}{r^{2}}\right) \mathrm{d} t^{2}-\frac{\mathrm{d} r^{2}}{r_{+}^{2}\left(1-\frac{r_{0}^{2}}{r^{2}}\right)}+r^{2}\left(\mathrm{~d} \varphi-\frac{r_{+} r_{0}}{r^{2}} \mathrm{~d} t\right)^{2}
$$


In the near horizon approximation, $r^{2}=r_{0}^{2}+2 r_{0} \rho$, we recover the line-element (B.5) with

$$
a=-\frac{r_{+}^{2}}{r_{0}} \quad \eta=-r_{+} \quad x=r_{0} \varphi .
$$

The cosmological temperature $T=r_{+}^{2} /\left(2 \pi r_{0}\right)$ again coincides with the Unruh temperature (B.2), up to a sign. This sign is explained by inner horizon black hole mechanics $[132,133]$.

The fact that Hawking/cosmological temperatures coincide with the Rindler temperature is not surprising but follows from kinematics. What is less clear is whether or not extensive quantities like free energy or entropy coincide as well. We calculate these quantities in the next two subsections.

\section{B.2 Rindler free energy}

Since we have no Rindler boundary conditions we do not know what the correct on-shell action is, as we have no way of checking the variational principle. However, since the zeromode solutions of quasi-Rindler holography coincide with the zero-mode solutions used in Rindler thermodynamics it is plausible that the action (2.20) can be used again. We base our discussion of free energy and entropy on this assumption.

Evaluating the full action (2.20) on-shell and multiplying it by temperature $T=\beta^{-1}$ yields the free energy,

$$
F=-\left.\frac{T}{8 \pi G_{N}} \int_{0}^{\tilde{L}} \mathrm{~d} y \int_{0}^{\beta} \mathrm{d} t_{\mathrm{E}} \sqrt{\gamma} K\right|_{\rho \rightarrow \infty}
$$

The quantity $\tilde{L}$ denotes the range of the coordinate $y$ and physically corresponds to the horizon area. If $\tilde{L}$ tends to infinity we simply define densities of extensive quantities like free energy or entropy by dividing all such expressions by $\tilde{L}$. Insertion of the boosted Rindler metric (4.26) into the general expression for free energy (B.10) yields

$$
F=-\frac{a \tilde{L}}{8 \pi G_{N}}=-\frac{T \tilde{L}}{4 G_{N}} .
$$

It is worthwhile mentioning that Rindler free energy (B.11) does not coincide with the corresponding BTZ or FSC free energy. Using the identifications (B.6) and (B.9) we find in both cases $F_{\mathrm{BTZ}}=F_{\mathrm{FSC}}=-T \tilde{L} /\left(8 G_{N}\right)$, which differs by a factor $1 / 2$ from the Rindler result (B.11). Nevertheless, as we shall demonstrate below, the corresponding entropies do coincide.

\section{B.3 Rindler entropy}

Our result for free energy (B.11) implies that Rindler entropy obeys the BekensteinHawking area law,

$$
S=-\frac{\mathrm{d} F}{\mathrm{~d} T}=\frac{\tilde{L}}{4 G_{N}}
$$


Note that entropy does not go to zero at arbitrarily small temperature. However, in that regime one should not trust the Rindler approximation since the $T \rightarrow 0$ limit is more adequately modelled by extremal horizons rather than non-extremal ones.

The result for entropy (B.12) can be obtained within the first order formulation as well. Applying the flat space results of $[44,45]$ to the present case yields

$$
S=\frac{k}{2 \pi} \int_{0}^{\beta} \mathrm{d} u \int_{0}^{\tilde{L}} \mathrm{~d} x\left\langle A_{u} A_{x}\right\rangle=\frac{k}{2 \pi} \tilde{L} \beta\left\langle\mathfrak{a}_{u} \mathfrak{a}_{x}\right\rangle=k \tilde{L} .
$$

Relating the Chern-Simons level with the inverse Newton constant, $k=1 /\left(4 G_{N}\right)$ then reproduces precisely the Bekenstein-Hawking area law (B.12).

Interestingly, Rindler entropy (B.12) also follows from near horizon BTZ entropy. The latter is given by

$$
S_{\mathrm{BTZ}}=\frac{2 \pi r_{+}}{4 G_{N}}=\frac{2 \pi \hat{r}_{+} \ell^{2}}{4 G_{N}}=\frac{\tilde{L}}{4 G_{N}} .
$$

In the last equality we identified the length of the $x$-interval using the last relation (B.6) together with $\varphi \sim \varphi+2 \pi$. Thus, the near horizon BTZ entropy coincides with the Rindler entropy, which provides another consistency check on the correctness of our result.

The same conclusions hold for the entropy of flat space cosmologies,

$$
S_{\mathrm{FSC}}=\frac{2 \pi r_{0}}{4 G_{N}}=\frac{\tilde{L}}{4 G_{N}} .
$$

In the last equality we identified the length of the $x$-interval using the last relation (B.9) together with $\varphi \sim \varphi+2 \pi$.

The results above confirm that entropy is a near-horizon property, whereas free energy and the conserved charges are a property of the global spacetime.

Open Access. This article is distributed under the terms of the Creative Commons Attribution License (CC-BY 4.0), which permits any use, distribution and reproduction in any medium, provided the original author(s) and source are credited.

\section{References}

[1] A. Staruszkiewicz, Gravitation Theory in Three-Dimensional Space, Acta Phys. Polon. 24 (1963) 735 [INSPIRE].

[2] S. Deser, R. Jackiw and G. 't Hooft, Three-Dimensional Einstein Gravity: Dynamics of Flat Space, Annals Phys. 152 (1984) 220 [inSPIRE].

[3] S. Deser and R. Jackiw, Three-Dimensional Cosmological Gravity: Dynamics of Constant Curvature, Annals Phys. 153 (1984) 405 [INSPIRE].

[4] M. Bañados, C. Teitelboim and J. Zanelli, The black hole in three-dimensional space-time, Phys. Rev. Lett. 69 (1992) 1849 [hep-th/9204099] [INSPIRE].

[5] M. Bañados, M. Henneaux, C. Teitelboim and J. Zanelli, Geometry of the (2+1) black hole, Phys. Rev. D 48 (1993) 1506 [Erratum ibid. D 88 (2013) 069902] [gr-qc/9302012] [INSPIRE]. 
[6] L. Cornalba and M.S. Costa, A new cosmological scenario in string theory, Phys. Rev. D 66 (2002) 066001 [hep-th/0203031] [INSPIRE].

[7] L. Cornalba and M.S. Costa, Time dependent orbifolds and string cosmology, Fortsch. Phys. 52 (2004) 145 [hep-th/0310099] [INSPIRE].

[8] J.D. Brown and M. Henneaux, Central Charges in the Canonical Realization of Asymptotic Symmetries: An Example from Three-Dimensional Gravity, Commun. Math. Phys. 104 (1986) 207 [INSPIRE].

[9] M. Henneaux, C. Martinez, R. Troncoso and J. Zanelli, Black holes and asymptotics of $2+1$ gravity coupled to a scalar field, Phys. Rev. D 65 (2002) 104007 [hep-th/0201170] [INSPIRE].

[10] D. Grumiller and N. Johansson, Consistent boundary conditions for cosmological topologically massive gravity at the chiral point, Int. J. Mod. Phys. D 17 (2009) 2367 [arXiv:0808.2575] [INSPIRE].

[11] M. Henneaux, C. Martinez and R. Troncoso, Asymptotically anti-de Sitter spacetimes in topologically massive gravity, Phys. Rev. D 79 (2009) 081502 [arXiv:0901.2874] [InSPIRE].

[12] G. Compère, W. Song and A. Strominger, New Boundary Conditions for AdS $S_{3}$, JHEP 05 (2013) 152 [arXiv:1303.2662] [INSPIRE].

[13] C. Troessaert, Enhanced asymptotic symmetry algebra of AdS $S_{3}$, JHEP 08 (2013) 044 [arXiv:1303.3296] [INSPIRE].

[14] G. Barnich and G. Compere, Classical central extension for asymptotic symmetries at null infinity in three spacetime dimensions, Class. Quant. Grav. 24 (2007) F15 [gr-qc/0610130] [INSPIRE].

[15] A. Ashtekar, J. Bicak and B.G. Schmidt, Asymptotic structure of symmetry reduced general relativity, Phys. Rev. D 55 (1997) 669 [gr-qc/9608042] [INSPIRE].

[16] A. Bagchi and R. Gopakumar, Galilean Conformal Algebras and AdS/CFT, JHEP 07 (2009) 037 [arXiv: 0902.1385] [InSPIRE].

[17] A. Bagchi, R. Gopakumar, I. Mandal and A. Miwa, GCA in 2d, JHEP 08 (2010) 004 [arXiv:0912.1090] [INSPIRE].

[18] A. Bagchi, The BMS/GCA correspondence, arXiv:1006.3354 [INSPIRE].

[19] A. Bagchi, S. Detournay and D. Grumiller, Flat-Space Chiral Gravity, Phys. Rev. Lett. 109 (2012) 151301 [arXiv:1208.1658] [inSPIRE].

[20] G. Barnich, Entropy of three-dimensional asymptotically flat cosmological solutions, JHEP 10 (2012) 095 [arXiv: 1208.4371] [INSPIRE].

[21] A. Bagchi, S. Detournay, R. Fareghbal and J. Simón, Holography of 3D Flat Cosmological Horizons, Phys. Rev. Lett. 110 (2013) 141302 [arXiv: 1208.4372] [INSPIRE].

[22] A. Bagchi, S. Detournay, D. Grumiller and J. Simón, Cosmic Evolution from Phase Transition of Three-Dimensional Flat Space, Phys. Rev. Lett. 111 (2013) 181301 [arXiv: 1305.2919] [INSPIRE].

[23] S. Detournay, D. Grumiller, F. Schöller and J. Simón, Variational principle and one-point functions in three-dimensional flat space Einstein gravity, Phys. Rev. D 89 (2014) 084061 [arXiv: 1402 .3687] [INSPIRE]. 
[24] H. Afshar, A. Bagchi, R. Fareghbal, D. Grumiller and J. Rosseel, Spin-3 Gravity in Three-Dimensional Flat Space, Phys. Rev. Lett. 111 (2013) 121603 [arXiv:1307.4768] [INSPIRE].

[25] H.A. Gonzalez, J. Matulich, M. Pino and R. Troncoso, Asymptotically flat spacetimes in three-dimensional higher spin gravity, JHEP 09 (2013) 016 [arXiv:1307.5651] [INSPIRE].

[26] G. Barnich and B. Oblak, Notes on the BMS group in three dimensions: I. Induced representations, JHEP 06 (2014) 129 [arXiv:1403.5803] [INSPIRE].

[27] G. Barnich and B. Oblak, Notes on the BMS group in three dimensions: II. Coadjoint representation, JHEP 03 (2015) 033 [arXiv:1502.00010] [INSPIRE].

[28] B. Oblak, Characters of the BMS Group in Three Dimensions, Commun. Math. Phys. 340 (2015) 413 [arXiv: 1502.03108] [INSPIRE].

[29] G. Barnich, H.A. Gonzalez, A. Maloney and B. Oblak, One-loop partition function of three-dimensional flat gravity, JHEP 04 (2015) 178 [arXiv: 1502.06185] [INSPIRE].

[30] A. Bagchi, R. Basu, D. Grumiller and M. Riegler, Entanglement entropy in Galilean conformal field theories and flat holography, Phys. Rev. Lett. 114 (2015) 111602 [arXiv: 1410.4089] [INSPIRE].

[31] S.M. Hosseini and A. Veliz-Osorio, Gravitational anomalies, entanglement entropy and flat-space holography, Phys. Rev. D 93 (2016) 046005 [arXiv:1507.06625] [INSPIRE].

[32] R. Basu and M. Riegler, Wilson Lines and Holographic Entanglement Entropy in Galilean Conformal Field Theories, Phys. Rev. D 93 (2016) 045003 [arXiv:1511.08662] [InSPIRE].

[33] G. Barnich, A. Gomberoff and H.A. González, The flat limit of three dimensional asymptotically anti-de Sitter spacetimes, Phys. Rev. D 86 (2012) 024020 [arXiv:1204.3288] [INSPIRE].

[34] A. Bagchi and R. Fareghbal, BMS/GCA Redux: Towards Flatspace Holography from Non-Relativistic Symmetries, JHEP 10 (2012) 092 [arXiv:1203.5795] [INSPIRE].

[35] A. Bagchi, Tensionless Strings and Galilean Conformal Algebra, JHEP 05 (2013) 141 [arXiv: 1303.0291] [INSPIRE].

[36] R.N. Caldeira Costa, Aspects of the zero $\Lambda$ limit in the AdS/CFT correspondence, Phys. Rev. D 90 (2014) 104018 [arXiv:1311.7339] [INSPIRE].

[37] R. Fareghbal and A. Naseh, Flat-Space Energy-Momentum Tensor from BMS/GCA Correspondence, JHEP 03 (2014) 005 [arXiv: 1312.2109] [INSPIRE].

[38] C. Krishnan, A. Raju and S. Roy, A Grassmann path from AdS $S_{3}$ to flat space, JHEP 03 (2014) 036 [arXiv: 1312.2941] [inSPIRE].

[39] A. Bagchi and R. Basu, 3D Flat Holography: Entropy and Logarithmic Corrections, JHEP 03 (2014) 020 [arXiv: 1312.5748] [INSPIRE].

[40] G. Barnich, L. Donnay, J. Matulich and R. Troncoso, Asymptotic symmetries and dynamics of three-dimensional flat supergravity, JHEP 08 (2014) 071 [arXiv: 1407.4275] [INSPIRE].

[41] M. Riegler, Flat space limit of higher-spin Cardy formula, Phys. Rev. D 91 (2015) 024044 [arXiv: 1408.6931] [INSPIRE].

[42] R. Fareghbal and A. Naseh, Aspects of Flat/CCFT Correspondence, Class. Quant. Grav. 32 (2015) 135013 [arXiv:1408.6932] [InSPIRE]. 
[43] R. Fareghbal and S.M. Hosseini, Holography of 3D Asymptotically Flat Black Holes, Phys. Rev. D 91 (2015) 084025 [arXiv: 1412.2569] [InSPIRE].

[44] M. Gary, D. Grumiller, M. Riegler and J. Rosseel, Flat space (higher spin) gravity with chemical potentials, JHEP 01 (2015) 152 [arXiv: 1411.3728] [INSPIRE].

[45] J. Matulich, A. Perez, D. Tempo and R. Troncoso, Higher spin extension of cosmological spacetimes in 3D: asymptotically flat behaviour with chemical potentials and thermodynamics, JHEP 05 (2015) 025 [arXiv: 1412.1464] [INSPIRE].

[46] A. Bagchi, D. Grumiller and W. Merbis, Stress tensor correlators in three-dimensional gravity, Phys. Rev. D 93 (2016) 061502 [arXiv:1507.05620] [InSPIRE].

[47] W. Rindler, Kruskal Space and the Uniformly Accelerated Frame, Am. J. Phys. 34 (1966) 1174 .

[48] S.A. Fulling, Nonuniqueness of canonical field quantization in Riemannian space-time, Phys. Rev. D 7 (1973) 2850 [inSPIRE].

[49] P.C.W. Davies, Scalar particle production in Schwarzschild and Rindler metrics, J. Phys. A 8 (1975) 609 [INSPIRE].

[50] W.G. Unruh, Notes on black hole evaporation, Phys. Rev. D 14 (1976) 870 [InSPIRE].

[51] W.G. Unruh and R.M. Wald, What happens when an accelerating observer detects a Rindler particle, Phys. Rev. D 29 (1984) 1047 [INSPIRE].

[52] R. Laflamme, Entropy of a Rindler Wedge, Phys. Lett. B 196 (1987) 449 [INSPIRE].

[53] D.A. Lowe and A. Strominger, Strings near a Rindler or black hole horizon, Phys. Rev. D 51 (1995) 1793 [hep-th/9410215] [INSPIRE].

[54] S. Deser and O. Levin, Accelerated detectors and temperature in (anti)-de Sitter spaces, Class. Quant. Grav. 14 (1997) L163 [gr-qc/9706018] [inSPIRE].

[55] S. Deser and O. Levin, Equivalence of Hawking and Unruh temperatures through flat space embeddings, Class. Quant. Grav. 15 (1998) L85 [hep-th/9806223] [INSPIRE].

[56] S. Deser and O. Levin, Mapping Hawking into Unruh thermal properties, Phys. Rev. D 59 (1999) 064004 [hep-th/9809159] [INSPIRE].

[57] S. Carlip, Entropy from conformal field theory at Killing horizons, Class. Quant. Grav. 16 (1999) 3327 [gr-qc/9906126] [INSPIRE].

[58] T. Padmanabhan, Thermodynamics and/of horizons: A comparison of Schwarzschild, Rindler and de Sitter space-times, Mod. Phys. Lett. A 17 (2002) 923 [gr-qc/0202078] [INSPIRE].

[59] V. Moretti and N. Pinamonti, Holography and $\mathrm{SL}(2, \mathbb{R})$ symmetry in $2 D$ Rindler space-time, J. Math. Phys. 45 (2004) 230 [hep-th/0304111] [INSPIRE].

[60] D. Marolf, D. Minic and S.F. Ross, Notes on space-time thermodynamics and the observer dependence of entropy, Phys. Rev. D 69 (2004) 064006 [hep-th/0310022] [INSPIRE].

[61] A.J. Amsel, D. Marolf and A. Virmani, The Physical Process First Law for Bifurcate Killing Horizons, Phys. Rev. D 77 (2008) 024011 [arXiv:0708.2738] [InSPIRE].

[62] H. Casini, Relative entropy and the Bekenstein bound, Class. Quant. Grav. 25 (2008) 205021 [arXiv: 0804.2182] [INSPIRE]. 
[63] H. Chung, Asymptotic Symmetries of Rindler Space at the Horizon and Null Infinity, Phys. Rev. D 82 (2010) 044019 [arXiv: 1005.0820] [InSPIRE].

[64] D. Grumiller, Model for gravity at large distances, Phys. Rev. Lett. 105 (2010) 211303 [Erratum ibid. 106 (2011) 039901] [arXiv:1011.3625] [INSPIRE].

[65] S. Carloni, D. Grumiller and F. Preis, Solar system constraints on Rindler acceleration, Phys. Rev. D 83 (2011) 124024 [arXiv:1103.0274] [InSPIRE].

[66] D. Grumiller, M. Irakleidou, I. Lovrekovic and R. McNees, Conformal gravity holography in four dimensions, Phys. Rev. Lett. 112 (2014) 111102 [arXiv:1310.0819] [InSPIRE].

[67] M. Parikh, P. Samantray and E. Verlinde, Rotating Rindler-AdS Space, Phys. Rev. D 86 (2012) 024005 [arXiv:1112.3433] [INSPIRE].

[68] B. Czech, J.L. Karczmarek, F. Nogueira and M. Van Raamsdonk, Rindler Quantum Gravity, Class. Quant. Grav. 29 (2012) 235025 [arXiv:1206.1323] [INSPIRE].

[69] M. Parikh and P. Samantray, Rindler-AdS/CFT, arXiv:1211.7370 [InSPIRE].

[70] T.G. Mertens, H. Verschelde and V.I. Zakharov, Random Walks in Rindler Spacetime and String Theory at the Tip of the Cigar, JHEP 03 (2014) 086 [arXiv:1307.3491] [INSPIRE].

[71] E. Halyo, Rindler Energy is Wald Entropy, arXiv:1403.2333 [INSPIRE].

[72] E. Halyo, On the Holographic Nature Of Rindler Energy, arXiv:1406.5763 [INSPIRE].

[73] E. Halyo, Black Holes as Conformal Field Theories on Horizons, arXiv:1502.01979 [INSPIRE].

[74] R. Fareghbal and A. Naseh, Rindler/Contracted-CFT Correspondence, JHEP 06 (2014) 134 [arXiv: 1404.3937] [INSPIRE].

[75] S. Carlip, Black hole entropy from horizon conformal field theory, Nucl. Phys. Proc. Suppl. 88 (2000) 10 [gr-qc/9912118] [INSPIRE].

[76] G. Compere, The Kerr/CFT correspondence and its extensions: a comprehensive review, Living Rev. Rel. 15 (2012) 11 [arXiv:1203.3561] [INSPIRE].

[77] A. Achucarro and P.K. Townsend, A Chern-Simons Action for Three-Dimensional anti-de Sitter Supergravity Theories, Phys. Lett. B 180 (1986) 89 [INSPIRE].

[78] E. Witten, (2+1)-Dimensional Gravity as an Exactly Soluble System, Nucl. Phys. B 311 (1988) 46 [INSPIRE].

[79] G. Barnich and H.A. Gonzalez, Dual dynamics of three dimensional asymptotically flat Einstein gravity at null infinity, JHEP 05 (2013) 016 [arXiv:1303.1075] [INSPIRE].

[80] H.R. Afshar, Flat/AdS boundary conditions in three dimensional conformal gravity, JHEP 10 (2013) 027 [arXiv: 1307.4855] [INSPIRE].

[81] J.W. York Jr., Role of conformal three geometry in the dynamics of gravitation, Phys. Rev. Lett. 28 (1972) 1082 [INSPIRE].

[82] G.W. Gibbons and S.W. Hawking, Action Integrals and Partition Functions in Quantum Gravity, Phys. Rev. D 15 (1977) 2752 [InSPIRE].

[83] M. Gary, D. Grumiller and R. Rashkov, Towards non-AdS holography in 3-dimensional higher spin gravity, JHEP 03 (2012) 022 [arXiv: 1201.0013] [INSPIRE]. 
[84] H. Afshar, M. Gary, D. Grumiller, R. Rashkov and M. Riegler, Non-AdS holography in 3-dimensional higher spin gravity - General recipe and example, JHEP 11 (2012) 099 [arXiv:1209.2860] [INSPIRE].

[85] J.M. Maldacena, The large $N$ limit of superconformal field theories and supergravity, Int. J. Theor. Phys. 38 (1999) 1113 [hep-th/9711200] [InSPIRE].

[86] S. Detournay, T. Hartman and D.M. Hofman, Warped Conformal Field Theory, Phys. Rev. D 86 (2012) 124018 [arXiv:1210.0539] [InSPIRE].

[87] G. Compere and S. Detournay, Semi-classical central charge in topologically massive gravity, Class. Quant. Grav. 26 (2009) 012001 [Erratum ibid. 26 (2009) 139801] [arXiv:0808.1911] [INSPIRE].

[88] G. Compere and S. Detournay, Boundary conditions for spacelike and timelike warped $A d S_{3}$ spaces in topologically massive gravity, JHEP 08 (2009) 092 [arXiv:0906.1243] [INSPIRE].

[89] M. Blagojevic and B. Cvetkovic, Asymptotic structure of topologically massive gravity in spacelike stretched AdS sector, JHEP 09 (2009) 006 [arXiv:0907.0950] [INSPIRE].

[90] M. Henneaux, C. Martinez and R. Troncoso, Asymptotically warped anti-de Sitter spacetimes in topologically massive gravity, Phys. Rev. D 84 (2011) 124016 [arXiv: 1108.2841] [INSPIRE].

[91] M. Bertin, S. Ertl, H. Ghorbani, D. Grumiller, N. Johansson and D. Vassilevich, Lobachevsky holography in conformal Chern-Simons gravity, JHEP 06 (2013) 015 [arXiv:1212.3335] [INSPIRE].

[92] H. Afshar, B. Cvetkovic, S. Ertl, D. Grumiller and N. Johansson, Holograms of Conformal Chern-Simons Gravity, Phys. Rev. D 84 (2011) 041502 [arXiv:1106.6299] [INSPIRE].

[93] H. Afshar, B. Cvetkovic, S. Ertl, D. Grumiller and N. Johansson, Conformal Chern-Simons holography - lock, stock and barrel, Phys. Rev. D 85 (2012) 064033 [arXiv:1110.5644] [INSPIRE].

[94] D.M. Hofman and B. Rollier, Warped Conformal Field Theory as Lower Spin Gravity, Nucl. Phys. B 897 (2015) 1 [arXiv:1411.0672] [InSPIRE].

[95] D.M. Hofman and A. Strominger, Chiral Scale and Conformal Invariance in $2 D$ Quantum Field Theory, Phys. Rev. Lett. 107 (2011) 161601 [arXiv:1107.2917] [INSPIRE].

[96] M. Bañados, Global charges in Chern-Simons field theory and the $(2+1)$ black hole, Phys. Rev. D 52 (1996) 5816 [hep-th/9405171] [INSPIRE].

[97] H. Afshar, A. Bagchi, S. Detournay, D. Grumiller, S. Prohazka and M. Riegler, Holographic Chern-Simons Theories, Lect. Notes Phys. 892 (2015) 311 [arXiv:1404.1919] [INSPIRE].

[98] T. Regge and C. Teitelboim, Role of Surface Integrals in the Hamiltonian Formulation of General Relativity, Annals Phys. 88 (1974) 286 [INSPIRE].

[99] G. Barnich and F. Brandt, Covariant theory of asymptotic symmetries, conservation laws and central charges, Nucl. Phys. B 633 (2002) 3 [hep-th/0111246] [InSPIRE].

[100] G. Barnich and G. Compere, Surface charge algebra in gauge theories and thermodynamic integrability, J. Math. Phys. 49 (2008) 042901 [arXiv:0708. 2378] [InSPIRE].

[101] A. Garbarz and M. Leston, Classification of Boundary Gravitons in AdS $S_{3}$ Gravity, JHEP 05 (2014) 141 [arXiv: 1403.3367] [INSPIRE]. 
[102] G. Barnich and B. Oblak, Holographic positive energy theorems in three-dimensional gravity, Class. Quant. Grav. 31 (2014) 152001 [arXiv:1403.3835] [INSPIRE].

[103] G. Compère, P.-J. Mao, A. Seraj and M.M. Sheikh-Jabbari, Symplectic and Killing symmetries of $A d S_{3}$ gravity: holographic vs boundary gravitons, JHEP 01 (2016) 080 [arXiv: 1511.06079] [INSPIRE].

[104] J. Unterberger and C. Roger, The Schrödinger-Virasoro Algebra: Mathematical structure and dynamical Schrödinger symmetries. Theoretical and Mathematical Physics, Springer, Berlin Heidelberg, (2011).

[105] N.T. Bishop, R. Gomez, L. Lehner, M. Maharaj and J. Winicour, High powered gravitational news, Phys. Rev. D 56 (1997) 6298 [gr-qc/9708065] [InSPIRE].

[106] E. Shaghoulian, A Cardy formula for holographic hyperscaling-violating theories, JHEP 11 (2015) 081 [arXiv: 1504.02094] [InSPIRE].

[107] L. Donnay, G. Giribet, H.A. González and M. Pino, Supertranslations and Superrotations at the Black Hole Horizon, Phys. Rev. Lett. 116 (2016) 091101 [arXiv:1511. 08687] [INSPIRE].

[108] . S.W. Hawking, The Information Paradox for Black Holes, arXiv:1509.01147 [INSPIRE].

[109] M.J. Perry, Black Hole Memory, talk delivered at the Nordic Institute for Theoretical Physics (NORDITA), Stockholm, Sweden, (2015).

[110] S.W. Hawking, M.J. Perry and A. Strominger, Soft Hair on Black Holes, arXiv: 1601.00921 [INSPIRE].

[111] M. Blau and M. O'Loughlin, Horizon Shells and BMS-like Soldering Transformations, JHEP 03 (2016) 029 [arXiv:1512.02858] [INSPIRE].

[112] E. Ayón-Beato and G. Velázquez-Rodríguez, Residual symmetries of the gravitational field, Phys. Rev. D 93 (2016) 044040 [arXiv:1511.07461] [inSPIRE].

[113] S. Deser, R. Jackiw and S. Templeton, Three-Dimensional Massive Gauge Theories, Phys. Rev. Lett. 48 (1982) 975 [inSPIRE].

[114] S. Deser, R. Jackiw and S. Templeton, Topologically Massive Gauge Theories, Annals Phys. 140 (1982) 372 [Erratum ibid. 185 (1988) 406] [INSPIRE].

[115] L. Guieu and C. Roger, L'algèbre et le groupe de Virasoro: aspects géométriques et algébriques, généralisations, les Publications CRM, (2007).

[116] C. Roger and J. Unterberger, The Schrödinger-Virasoro Lie group and algebra: From geometry to representation theory, Annales Henri Poincaré 7 (2006) 1477 [math-ph/0601050] [INSPIRE].

[117] E. Witten, Coadjoint Orbits of the Virasoro Group, Commun. Math. Phys. 114 (1988) 1 [INSPIRE].

[118] S. Weinberg, The Quantum Theory of Fields, volume I, Cambridge University Press, (1995).

[119] G. Mackey, Infinite dimensional group representations, Bull. Amer. Math. Soc. 69 (1963) 628.

[120] G. Mackey, Induced representations of groups and quantum mechanics, Publicazioni della Classe di Scienze della Scuola Normale Superiore di Pisa, W.A. Benjamin, (1968).

[121] Infinite Dimensional Group Representations and Their Applications. Forschungsinstitut für Mathematik, ETH, (1971). 
[122] E.P. Wigner, On unitary representations of the inhomogeneous Lorentz group, Annals Math. 40 (1939) 149 [Nucl. Phys. Proc. Suppl. 6 (1989) 9].

[123] J. Cornwell, Group theory in physics, Techniques of physics, Academic Press, (1984).

[124] A. Barut and R. Raczka, Theory of Group Representations and Applications, World Scientific, (1986).

[125] E.P. Wigner, Unitary representations of the inhomogeneous Lorentz group including reflections, in Group theoretical concepts and methods in elementary particle physics, Lectures of the Istanbul summer school of theoretical physics (1962), F. Gürsey ed., Gordon and Breach, New York, U.S.A. (1964), pp. 37-80.

[126] E. Shavgulidze, A measure that is quasi-invariant with respect to the action of a group of diffeomorphisms of a finite-dimensional manifold, Dokl. Akad. Nauk SSSR 303 (1988) 811.

[127] E. Shavgulidze, An example of a measure quasi-invariant under the action of the diffeomorphism group of the circle, Funkts. Anal. Prilozh. 12 (1978) 55.

[128] E. Shavgulidze, Mesures quasi-invariantes sur les groupes de difféomorphismes des variétés riemaniennes, C. R. Acad. Sci. Paris 321 (1995) 229.

[129] E. Shavgulidze, Quasiinvariant measures on groups of diffeomorphisms, in Loop spaces and groups of diffeomorphisms. Collected papers, MAIK Nauka/Interperiodica Publishing, Moscow, Russia (1997), pp. 181-202, [Tr. Mat. Inst. Steklova 217 (1997) 189].

[130] S. Giombi, A. Maloney and X. Yin, One-loop Partition Functions of 3D Gravity, JHEP 08 (2008) 007 [arXiv:0804.1773] [INSPIRE].

[131] A. Bagchi and I. Mandal, On Representations and Correlation Functions of Galilean Conformal Algebras, Phys. Lett. B 675 (2009) 393 [arXiv:0903.4524] [InSPIRE].

[132] A. Castro and M.J. Rodriguez, Universal properties and the first law of black hole inner mechanics, Phys. Rev. D 86 (2012) 024008 [arXiv:1204.1284] [INSPIRE].

[133] S. Detournay, Inner Mechanics of 3d Black Holes, Phys. Rev. Lett. 109 (2012) 031101 [arXiv: 1204.6088] [INSPIRE]. 